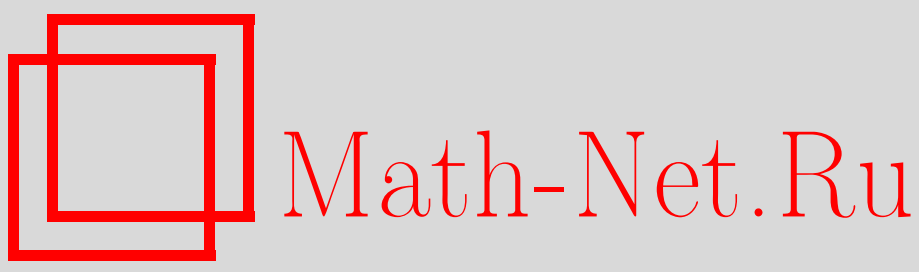

А. Ю. Колесов, Н. Х. Розов, Влияние квадратичной нелинейности на динамику периодических решений волнового уравнения, Матем. сб., 2002, том 193, номер 1, 93 118

DOI: https://doi.org/10.4213/sm622

Использование Общероссийского математического портала Math-Net.Ru подразумевает, что вы прочитали и согласны с пользовательским соглашением

http://www.mathnet.ru/rus/agreement

Параметры загрузки:

IP: 18.208 .226 .222

26 апреля 2023 г., $12: 38: 48$ 


\title{
Влияние квадратичной нелинейности на динамику периодических решений волнового уравнения
}

\begin{abstract}
Для нелинейного телеграффного уравнения с нулевьми граничньми условиями Дирихле или Неймана на концах конечного отрезка рассматривается вопрос о существовании и устойчивости периодических по времени решений, бифурцирующих из нулевого положения равновесия. Изучается динамика этих решений при изменении коэффициента диффузии (т.е. коэффициента перед второй производной по пространственной переменной). В случаеграничных условий Дирихле показьвается существенная ее зависимость от наличия или отсутствия в нелинейности квадратичных слагаемых. Точнее говоря, устанавливается, что квадратичная нелинейность приводит к появлению бесконечной последовательности бифуркаций, происходящих с каждым из периодических решений при неограниченном уменьшении диффузии. Попутно исследуется связанный с данньм эфффектом вопрос о границе применимости метода квазинормальных форм Ю. С. Колесова для построения автоколебаний у сингулярно возмущенных гиперболических краевых задач.
\end{abstract}

Библиограбия: 19 названий.

\section{Введение}

Проблема аттракторов гиперболических краевых задач берет свое начало с исследования автоколебаний в линейных системах телеграфыных уравнений с нелинейными граничными условиями на концах конечного отрезка. Изучение такого типа задач актуально в связи с тем, что они служат математическими моделями для широкого класса автогенераторов с отрезком длинной линии в цепи обратной связи. В качестве примера сошлемся на классическую работу А.А. Витта [1], являющуюся, по всей видимости, первой публикацией, посвященной данному вопросу. Напомним, что объектом анализа в [1] была краевая задача указанного типа, представляюшая собой модель двухпроводной линии, к одной стороне которой присоединена емкость, а к другой - нелинейное сопротивление с падающей характеристикой.

С помощью известного в физической теории колебаний метода медленно меняюшихся фаз и амплитуд в [1] были получены асимптотические формулы для периодических по времени решений рассматриваемой краевой задачи и установлены условия устойчивости таких решений. Впоследствии упомянутые результаты переизлагались в ряде публикаций (см., например, [2], [3]), однако их строгое математическое обоснование дано лишь недавно [4]. Связано это с тем, что долгое время отсутствовал адекватный математический аппарат анализа автоколебаний распределенных систем в окрестности бесконечномерного вырождения. Создан же он был только в 80-е годы Ю. С. Колесовым сначала для параболических уравнений с мальми коэффициентами диффузии [5]-[7], а затем перенесен на аналогичный 
класс волновых уравнений [8], [9]. Дальнейшее развитие методов работ [5]-[7] применительно к гиперболическим уравнениям отражено в монографии [10].

В настоящей работе рассматривается вопрос о бифуркации автоколебаний у нелинейного волнового уравнения вида

$$
u_{t t}-\varepsilon u_{t}+u=a^{2} u_{x x}+f\left(u, u_{t}\right)
$$

с нулевыми граничными условиями Дирихле или Неймана на отрезке. Здесь $0<$ $\varepsilon \ll 1, a=\mathrm{const}>0$, а тейлоровское разложение в нуле функции $f(u, v) \in C^{\infty}$ начинается с квадратичных слагаемых.

В случае граничных условий Дирихле для уравнения (*) устанавливается новый феномен, не наблюдающийся при граничных условиях Неймана. А именно, показывается, что динамика периодических решений этого уравнения по параметру $а$ существенно зависит от наличия или отсутствия в тейлоровском разложении нелинейности $f(u, v)$ квадратичных слагаемых. Попутно обсуждается связанньй с данным эффектом вопрос о границе применимости метода квазинормальных форм Ю.С. Колесова (см. [8], [9]) для построения автоколебаний уравнения (*) при условии $a^{2}=\varepsilon \sigma^{2}, \sigma=$ const $>0$.

\section{§1. Общие свойства рассматриваемого уравнения}

1.1. Описание алгоритмов. Начнем с изучения для уравнения $(*)$ на отрезке $0 \leqslant x \leqslant \pi$ краевой задачи

$$
u_{t t}-\varepsilon u_{t}+u=a^{2} u_{x x}+f\left(u, u_{t}\right),\left.\quad u\right|_{x=0}=\left.u\right|_{x=\pi}=0 .
$$

В качестве фазового пространства (пространства начальных условий $\left.\left(u, u_{t}\right)\right)$ задачи (1.1) возьмем $\stackrel{\circ}{W} \underset{2}{2} \times \stackrel{\circ}{W} \underset{2}{1}$, где $\stackrel{\circ}{W} \underset{2}{j}, j=1,2,-$ соболевские пространства функций, удовлетворяющих граничным условиям из (1.1), и будем интересоваться ее аттракторами в указанном пространстве. Точнее говоря, поставим вопрос о существовании и устойчивости ее периодических по $t$ решений, бифурцирующих из нулевого положения равновесия при $\varepsilon>0$.

Пусть сначала $\varepsilon=0$. Тогда, как нетрудно увидеть, линеаризованная в нуле задача (1.1) допускает тригонометрические решения

$$
u=\exp \left( \pm i \omega_{n} t\right) \sin n x, \quad \omega_{n}=\sqrt{1+a^{2} n^{2}}, \quad n=1,2, \ldots
$$

Поэтому фиксируем произвольно натуральное $n$ и для построения асимптотики периодического решения, бифурцируюшего при $\varepsilon>0$ на моде $\sin n x$, воспользуемся стандартным одночастотньм методом из [11]. А именно, в данном случае, обыгрывая сушествование периодических решений (1.2), положим в (1.1)

$$
u=\varepsilon^{1 / 2} u_{1}(\tau, x)+\varepsilon u_{2}(\tau, x)+\varepsilon^{3 / 2} u_{3}(\tau, x)+\varepsilon^{2} u_{4}(\tau, x)+\cdots,
$$

где

$$
u_{1}=\left[\exp \left(i \omega_{n} \tau\right)+\exp \left(-i \omega_{n} \tau\right)\right] \xi_{n} \sin n x, \quad \tau=\left(1+\varepsilon \alpha_{n}+\cdots\right) t
$$


а $\xi_{n}, \alpha_{n}$ - некоторые неизвестные (подлежащие определению) вещественные постоянные. Привлекая затем тейлоровское разложение

$$
f(u, v)=a_{1} u^{2}+a_{2} u v+a_{3} v^{2}+b_{1} u^{3}+b_{2} u^{2} v+b_{3} u v^{2}+b_{4} v^{3}+\cdots
$$

и приравнивая коэффициенты при одинаковых степенях $\varepsilon$, для определения $u_{k}$, $k=2,3,4$, получим рекуррентную последовательность линейных неоднородных краевых задач

$$
\left(\frac{\partial^{2}}{\partial \tau^{2}}-a^{2} \frac{\partial^{2}}{\partial x^{2}}\right) u_{k}+u_{k}=g_{k}(\tau, x),\left.\quad u_{k}\right|_{x=0}=\left.u_{k}\right|_{x=\pi}=0
$$

Решения последних ишем в виде тригонометрических полиномов переменной $\omega_{n} \tau$ той же структуры, что и соответствующая неоднородность.

Несложные вычисления показывают, что

$$
u_{2}=\xi_{n}^{2}\left[A(x)+B(x) \exp \left(2 i \omega_{n} \tau\right)+\bar{B}(x) \exp \left(-2 i \omega_{n} \tau\right)\right]
$$

где коэффициенты $A(x), B(x)$ - решения краевых задач

$$
\begin{gathered}
A-a^{2} A^{\prime \prime}=2\left(a_{1}+\omega_{n}^{2} a_{3}\right) \sin ^{2} n x, \quad A(0)=A(\pi)=0 ; \\
\left(1-4 \omega_{n}^{2}\right) B-a^{2} B^{\prime \prime}=\left(a_{1}+i \omega_{n} a_{2}-\omega_{n}^{2} a_{3}\right) \sin ^{2} n x, \quad B(0)=B(\pi)=0 .
\end{gathered}
$$

Их анализ приводит, в свою очередь, к равенствам

$$
\begin{aligned}
& A(x)=\left(a_{1}+\omega_{n}^{2} a_{3}\right)\left[1-\frac{1}{\omega_{2 n}^{2}} \cos 2 n x-\left(1-\frac{1}{\omega_{2 n}^{2}}\right) \theta_{*}(x)\right] \\
& B(x)=\frac{1}{2}\left(a_{1}+i \omega_{n} a_{2}-\omega_{n}^{2} a_{3}\right)\left[\frac{1}{1-4 \omega_{n}^{2}}+\frac{1}{3} \cos 2 n x-\left(\frac{1}{3}+\frac{1}{1-4 \omega_{n}^{2}}\right) \theta_{* *}(x)\right]
\end{aligned}
$$

в которых

$$
\begin{gathered}
\theta_{*}(x)=\frac{[\operatorname{sh}(x / a)+\operatorname{sh}((\pi-x) / a)]}{\operatorname{sh}(\pi / a)}, \quad \theta_{* *}(x)=\frac{\cos \left[\sigma_{n}(x-\pi / 2)\right]}{\cos \left[\pi \sigma_{n} / 2\right]}, \\
\sigma_{n}=\sqrt{4 n^{2}+3 / a^{2}}
\end{gathered}
$$

Следует, однако, заметить, что если первая из задач (1.8) однозначно разрешима при любом значении параметра $a$, то функция $B(x)$ определяется формулами $(1.9),(1.10)$ только в том случае, когда $2 \omega_{n} \neq \omega_{2 k+1}, k=1,2, \ldots$, или, что то же самое, когда при всех натуральных $k, k \geqslant n$, справедливы неравенства

$$
a \neq a_{k}^{*}=\sqrt{3 /\left[(2 k+1)^{2}-4 n^{2}\right]}
$$

(в противном случае обращается в нуль знаменатель во второй формуле (1.10)). Поэтому условия (1.11) всюду ниже будем считать выполненньми.

На следуюшем шаге, т.е. при анализе краевой задачи (1.6) для $k=3$, учтем в ее правой части

$$
\begin{aligned}
g_{3}(\tau, x)= & \frac{\partial u_{1}}{\partial \tau}-2 \alpha_{n} \frac{\partial^{2} u_{1}}{\partial \tau^{2}}+2 a_{1} u_{1} u_{2}+a_{2} \frac{\partial}{\partial \tau}\left(u_{1} u_{2}\right)+2 a_{3} \frac{\partial u_{1}}{\partial \tau} \frac{\partial u_{2}}{\partial \tau}+b_{1} u_{1}^{3} \\
& +b_{2} u_{1}^{2} \frac{\partial u_{1}}{\partial \tau}+b_{3} u_{1}\left(\frac{\partial u_{1}}{\partial \tau}\right)^{2}+b_{4}\left(\frac{\partial u_{1}}{\partial \tau}\right)^{3}
\end{aligned}
$$


явный вид (1.4) и (1.7), (1.9), (1.10) функций $u_{1}, u_{2}$. В итоге убеждаемся, что решение $u_{3}$ задается равенством

$$
\begin{aligned}
u_{3}= & C(x) \exp \left(i \omega_{n} \tau\right)+\bar{C}(x) \exp \left(-i \omega_{n} \tau\right) \\
& +D(x) \exp \left(3 i \omega_{n} \tau\right)+\bar{D}(x) \exp \left(-3 i \omega_{n} \tau\right)
\end{aligned}
$$

где коэффициенты $C(x), D(x)$ определяются из краевых задач

$$
\begin{aligned}
& -a^{2}\left(C^{\prime \prime}+n^{2} C\right)=\varphi_{1}(x), \quad C(0)=C(\pi)=0 ; \\
& \left(1-9 \omega_{n}^{2}\right) D-a^{2} D^{\prime \prime}=\varphi_{2}(x), \quad D(0)=D(\pi)=0 \text {. }
\end{aligned}
$$

Здесь

$$
\begin{aligned}
\varphi_{1}= & \xi_{n}\left(i \omega_{n}+2 \alpha_{n} \omega_{n}^{2}\right) \sin n x+\xi_{n}^{3}\left[\left(2 a_{1}+i \omega_{n} a_{2}\right)(A(x)+B(x)) \sin n x\right. \\
& \left.+4 \omega_{n}^{2} a_{3} B(x) \sin n x+\left(3 b_{1}+i \omega_{n} b_{2}+\omega_{n}^{2} b_{3}+3 \omega_{n}^{3} i b_{4}\right) \sin ^{3} n x\right] \\
\varphi_{2}= & -\left(\frac{1}{3}+\frac{1}{1-4 \omega_{n}^{2}}\right)\left(2 a_{1}+3 i \omega_{n} a_{2}-4 \omega_{n}^{2} a_{3}\right) \xi_{n}^{3} \theta_{* *}(x) \sin n x+\varphi_{2}^{0}(x),
\end{aligned}
$$

а $\varphi_{2}^{0}(x)$ - некоторая линейная комбинация функций $\sin n x, \sin 3 n x$, явньй вид которой в дальнейшем не потребуется.

Обратимся сначала к первой из краевых задач (1.13) и заметим, что она вырождена (т.е. соответствующая однородная задача имеет нетривиальное решение). Условие же ее разрешимости

$$
\int_{0}^{\pi} \varphi_{1}(x) \sin n x d x=0
$$

будем рассматривать как уравнение для определения неизвестных постоянных $\xi_{n}, \alpha_{n}$. Учитывая, далее, в этом равенстве явный вид (1.14) неоднородности $\varphi_{1}(x)$, приходим к так называемому уравнению разветвления

$$
\left(\omega_{n}-2 i \alpha_{n} \omega_{n}^{2}\right) \xi_{n}+d_{n} \xi_{n}^{3}=0 .
$$

Здесь ляпуновская величина $d_{n}$ задается формулой

$$
\begin{aligned}
d_{n}= & d_{n}^{0}-\left(\beta_{1}-2 i \beta_{2}\right)\left(1-\frac{1}{\omega_{2 n}^{2}}\right) \frac{4 n^{2} a^{2}}{1+4 n^{2} a^{2}} \Delta_{1}\left(\frac{\pi}{2 a}\right) \\
& +\frac{2}{3}\left(3 \beta_{1}-2 i \beta_{3}\right)\left(\frac{1}{3}-\frac{1}{4 \omega_{n}^{2}-1}\right) a^{2} n^{2} \Delta_{2}\left(\frac{\pi}{2} \sigma_{n}\right),
\end{aligned}
$$

в которой

$$
\begin{gathered}
d_{n}^{0}=\beta_{1}\left(\frac{3}{4}+\frac{1}{2 \omega_{2 n}^{2}}-\frac{3}{2\left(4 \omega_{n}^{2}-1\right)}\right)+\frac{3}{4}\left(\omega_{n} b_{2}+3 \omega_{n}^{3} b_{4}\right) \\
-2 i \beta_{2}\left(1+\frac{1}{2 \omega_{2 n}^{2}}\right)-\frac{3}{4} i\left(3 b_{1}+\omega_{n}^{2} b_{3}\right)+i \beta_{3}\left(\frac{1}{6}+\frac{1}{4 \omega_{n}^{2}-1}\right), \\
\beta_{1}=\omega_{n} a_{2}\left(a_{1}+\omega_{n}^{2} a_{3}\right), \quad \beta_{2}=a_{1}\left(a_{1}+\omega_{n}^{2} a_{3}\right), \\
\beta_{3}=\left(a_{1}-\omega_{n}^{2} a_{3}\right)\left(a_{1}+2 \omega_{n}^{2} a_{3}\right)-a_{2}^{2} \omega_{n}^{2} / 2,
\end{gathered}
$$


а через $\Delta_{j}=\Delta_{j}(s), j=1,2$, обозначены соответственно функции $\operatorname{th}(s) / s \operatorname{tg}(s) / s$.

Предположим, что

$$
\operatorname{Re} d_{n}<0
$$

Тогда из (1.16) находим

$$
\xi_{n}=\sqrt{-\omega_{n} / \operatorname{Re} d_{n}}, \quad \alpha_{n}=\xi_{n}^{2} \operatorname{Im} d_{n} /\left(2 \omega_{n}^{2}\right),
$$

а затем, подставляя эти равенства в неоднородности (1.14), (1.15) и решая получившуюся краевую задачу для $C(x)$, полностью определяем первые два слагаемых в правой части формулы (1.12). Что же касается второй краевой задачи (1.13), то в силу (1.15) ее решение допускает, очевидно, представление:

$$
D(x)=-\left(\frac{1}{3}+\frac{1}{1-4 \omega_{n}^{2}}\right)\left(2 a_{1}+3 i \omega_{n} a_{2}-4 \omega_{n}^{2} a_{3}\right) \xi_{n}^{3} D_{1}(x)+D_{2}(x),
$$

где функция $D_{2}(x)$ однозначно определяется по $\varphi_{2}^{0}(x)$ и имеет ту же структуру, а $D_{1}(x)$ - решение краевой задачи

$$
\left(1-9 \omega_{n}^{2}\right) D_{1}-a^{2} D_{1}^{\prime \prime}=\theta_{* *}(x) \sin n x, \quad D_{1}(0)=D_{1}(\pi)=0 .
$$

Последняя же разрешима в том и только том случае, когда при всех натуральных $m, m \geqslant 3 n+1$, справедливы неравенства

$$
3 \omega_{n} \neq \omega_{m}
$$

Завершающий этап, т.е. анализ краевой задачи (1.6) при $k=4$, аналогичен случаю $k=2$. Действительно, здесь функцию $u_{4}$ следует искать в виде линейной комбинации гармоник $\exp \left( \pm i p \omega_{n} \tau\right), p=0,2,4$. Однозначную же разрешимость аналогичных (1.8) линейных неоднородных краевых задач для коэффициентов при указанных гармониках наряду с неравенствами (1.11) обеспечивают условия

$$
2 \omega_{n} \neq \omega_{2 k}, \quad 4 \omega_{n} \neq \omega_{k}, \quad k=1,2, \ldots,
$$

которые всюду ниже также считаем выполненными.

Итак, при условиях (1.11), (1.20), (1.22), (1.23) приближенное периодическое решение (1.3) может быть построено с точностью до $\varepsilon^{5 / 2}$ по невязке. Линеаризуя на нем уравнение $(*)$, получаем краевую задачу

$$
\begin{gathered}
h_{t t}-a^{2} h_{x x}+h=\sqrt{\varepsilon}\left[a_{1}(\tau, x) h+a_{2}(\tau, x) h_{t}\right]+\varepsilon\left[b_{1}(\tau, x, \varepsilon) h+b_{2}(\tau, x, \varepsilon) h_{t}\right], \\
\left.h\right|_{x=0}=\left.h\right|_{x=\pi}=0
\end{gathered}
$$

с $2 \pi / \omega_{n}$-периодическими по $\tau=\left(1+\varepsilon \alpha_{n}\right) t$ коэффициентами, в которой

$$
\begin{gathered}
a_{1}(\tau, x)=2 a_{1} u_{1}+a_{2} \frac{\partial u_{1}}{\partial \tau}, \quad a_{2}(\tau, x)=a_{2} u_{1}+2 a_{3} \frac{\partial u_{1}}{\partial \tau} \\
b_{1}(\tau, x, 0)=2 a_{1} u_{2}+a_{2} \frac{\partial u_{2}}{\partial \tau}+3 b_{1} u_{1}^{2}+2 b_{2} u_{1} \frac{\partial u_{1}}{\partial \tau}+b_{3}\left(\frac{\partial u_{1}}{\partial \tau}\right)^{2} \\
b_{2}(\tau, x, 0)=1+a_{2} u_{2}+2 a_{3} \frac{\partial u_{2}}{\partial \tau}+b_{2} u_{1}^{2}+2 b_{3} u_{1} \frac{\partial u_{1}}{\partial \tau}+3 b_{4}\left(\frac{\partial u_{1}}{\partial \tau}\right)^{2} .
\end{gathered}
$$


Как известно [12], основной этап доказательства существования у краевой задачи (1.1) цикла с асимптотикой (1.3) состоит в исследовании свойств устойчивости решений уравнения (1.24) и в обратимости соответствующего ему дифференциального оператора (в подходящем пространстве периодических функций). Обе эти проблемы решаются с помошью алгоритма исследования устойчивости из [13], адаптированного в [14] для телеграфных уравнений. Описание указанного алгоритма приводится ниже.

Начнем с расчета характеристических показателей (деленных на период логарифмов мультипликаторов) уравнения (1.24), отвечающих генерируемой моде $\sin n x$. В соответствии с развитой в [13] методикой положим в (1.24)

$$
\begin{gathered}
h=\left[V_{0}(\tau, x)+\sqrt{\varepsilon} V_{1}(\tau, x)+\varepsilon V_{2}(\tau, x)\right] \exp (\varepsilon D t), \\
V_{j}=\left[w_{j}, \overline{w_{j}}\right], \quad j=0,1,2, \quad w_{0}=\exp \left(i \omega_{n} \tau\right) \sin n x
\end{gathered}
$$

где $w_{j}$ - тригонометрические полиномы переменной $\omega_{n} \tau$, а матрица $D$ имеет структуру

$$
\left(\begin{array}{ll}
\varkappa_{1} & \varkappa_{2} \\
\varkappa_{2} & \varkappa_{1}
\end{array}\right) \text {. }
$$

Приравнивая коэффициенты при $\varepsilon^{1 / 2}$ и $\varepsilon$, для определения $w_{1}$ и $w_{2}$ получаем краевые задачи вида (1.6). В силу предполагаемых условий (1.11) первая из этих задач разрешима в нужном классе функций, а ее решение задается равенством

$$
w_{1}=\xi_{n}\left[A(x)+2 B(x) \exp \left(2 i \omega_{n} \tau\right)\right]
$$

где коэффициенты $A(x), B(x)$ те же, что и в (1.7). Далее, неоднородность в уравнении для $w_{2}$ представляет собой линейную комбинацию гармоник $\exp \left( \pm i p \omega_{n} \tau\right), p=1,3$. Поэтому, как и в случае задачи (1.6) при $k=3$, функцию $w_{2}$ ищем в виде $(1.12)$. Опуская несложные вычисления, аналогичные описанньм выше, отметим, что разрешимость краевых задач для коэффициентов этой функции при $\exp \left( \pm i \omega_{n} \tau\right)$ обеспечивается выбором неизвестных элементов $\varkappa_{1}$ и $\varkappa_{2}$ матрицы $(1.28)$, а краевые задачи для коэффициентов при $\exp \left( \pm 3 i \omega_{n} \tau\right)$ разрешимы в силу условий (1.22). При этом для $\varkappa_{1}, \varkappa_{2}$ выходят равенства

$$
\varkappa_{1}=d_{n} \xi_{n}^{2} /\left(2 \omega_{n}\right), \quad \varkappa_{2}=\varkappa_{1}
$$

Для расчета характеристических показателей краевой задачи (1.24) на модах $\sin m x, m=1,2, \ldots, m \neq n$, подставим в (1.24) представление

$$
h=\left[\exp \left(i \omega_{m} t\right) \sin m x+\varepsilon^{1 / 2} h_{1 m}(t, \tau, x)+\varepsilon h_{2 m}(t, \tau, x)\right] \exp \left(\varepsilon \mu_{m} t\right),
$$

где $h_{1 m}, h_{2 m}$ - тригонометрические многочлены переменных $\omega_{m} t, \omega_{n} \tau$, a $\mu_{m}-$ подлежащая определению комплексная постоянная. В результате, приравнивая коэффициенты при $\varepsilon^{1 / 2}$ и $\varepsilon$, для $h_{j m}, j=1,2$, получаем краевые задачи

$$
\begin{gathered}
\left(\frac{\partial^{2}}{\partial t^{2}}+2 \frac{\partial^{2}}{\partial t \partial \tau}+\frac{\partial^{2}}{\partial \tau^{2}}-a^{2} \frac{\partial^{2}}{\partial x^{2}}\right) h_{j m}+h_{j m}=f_{j}(t, \tau, x), \\
\left.h_{j m}\right|_{x=0}=\left.h_{j m}\right|_{x=\pi}=0,
\end{gathered}
$$


решения которых будем искать в виде тригонометрических полиномов той же структуры, что и соответствующие неоднородности.

При анализе краевых задач (1.31) нам потребуются специальные функции

$$
\theta_{*, m}^{ \pm, p}(x)=\sin \left(\sigma_{m, p}^{ \pm} x\right) / \sin \left(\pi \sigma_{m, p}^{ \pm}\right), \quad m=1,2, \ldots, \quad m \neq n, \quad p=1,2
$$

где частоты $\sigma_{m, p}^{ \pm}$задаются равенствами

$$
\sigma_{m, p}^{ \pm}=\frac{1}{a} \sqrt{\left(\omega_{m} \pm p \omega_{n}\right)^{2}-1}
$$

Подчеркнем, что подкоренные выражения в (1.33) могут быть как положительными, так и отрицательными. В случае же, если $\left(\omega_{m}-p \omega_{n}\right)^{2}=1$, считаем, что $\theta_{*, m}^{-, p}(x)=x / \pi$. Кроме того, будем предполагать выполнение при всех $m \neq n$ и $k=1,2, \ldots$ неравенств

$$
\left(\omega_{m} \pm p \omega_{n}\right)^{2}-\omega_{k}^{2} \neq 0, \quad p \omega_{n} \neq a k, \quad p=1,2
$$

обеспечивающих отличие от нуля знаменателей в (1.32) и стремление их модулей к ненулевьм конечным пределам при $m \rightarrow \infty$.

Обратимся теперь к краевой задаче (1.31) при $j=1$. Учитывая в ее правой части

$$
f_{1}(t, \tau, x)=\left[a_{1}(\tau, x)+i \omega_{m} a_{2}(\tau, x)\right] \exp \left(i \omega_{m} t\right) \sin m x
$$

равенства (1.25), приходим к выводу, что $h_{1 m}$ имеет вид:

$$
h_{1 m}=\exp \left[i\left(\omega_{m} t+\omega_{n} \tau\right)\right] C_{m}^{+}(x)+\exp \left[i\left(\omega_{m} t-\omega_{n} \tau\right)\right] C_{m}^{-}(x)
$$

где

$$
\begin{gathered}
C_{m}^{ \pm}(x)=v_{1 m}^{ \pm} \cos (m-n) x-v_{2 m}^{ \pm} \cos (m+n) x-\left(v_{1 m}^{ \pm}-v_{2 m}^{ \pm}\right) \theta_{*, m}^{ \pm, 1}(\pi-x) \\
-(-1)^{m+n}\left(v_{1 m}^{ \pm}-v_{2 m}^{ \pm}\right) \theta_{*, m}^{ \pm, 1}(x) \\
v_{1 m}^{ \pm}=v_{ \pm} /\left[\omega_{m-n}^{2}-\left(\omega_{m} \pm \omega_{n}\right)^{2}\right], \quad v_{2 m}^{ \pm}=v_{ \pm} /\left[\omega_{m+n}^{2}-\left(\omega_{m} \pm \omega_{n}\right)^{2}\right], \\
v_{ \pm}=\left(\xi_{n} / 2\right)\left[2 a_{1}+i\left(\omega_{m} \pm \omega_{n}\right) a_{2} \mp 2 \omega_{n} \omega_{m} a_{3}\right] .
\end{gathered}
$$

Отметим, что в (1.37) при любом $a>0$

$$
\left(\omega_{m}+\omega_{n}\right)^{2}-\omega_{m \pm n}^{2} \neq 0, \quad\left(\omega_{m}-\omega_{n}\right)^{2}-\omega_{m \pm n}^{2} \neq 0
$$

Действительно, в справедливости первого неравенства (1.39) убеждаемся, переписывая его в эквивалентной форме

$$
-1 / 2 \pm a^{2} m n \neq \sqrt{1+a^{2} n^{2}} \sqrt{1+a^{2} m^{2}}
$$

и замечая, что в силу неравенства Буняковского-Коши

$$
\left|-1 / 2 \pm a^{2} m n\right| \leqslant \sqrt{1 / 4+a^{2} n^{2}} \sqrt{1+a^{2} m^{2}}<\sqrt{1+a^{2} n^{2}} \sqrt{1+a^{2} m^{2}}
$$


Второе из неравенств (1.39) устанавливается аналогично. Отметим также, что при $m \rightarrow \infty$ коэффициенты (1.37) стремятся к конечным пределам, а значит,

$$
\left|v_{j m}^{ \pm}\right| \leqslant N, \quad j=1,2
$$

здесь и ниже через $N, N_{1}$ и т. д. обозначаем различные универсальные (не зависящие от $m, \varepsilon$ ) положительные постоянные, точные значения которых несущественны.

При анализе краевой задачи (1.31) в случае $j=2$ учтем в представлении

$$
\begin{aligned}
f_{2}(t, \tau, x)= & a_{1}(\tau, x) h_{1 m}+a_{2}(\tau, x)\left(\frac{\partial h_{1 m}}{\partial t}+\frac{\partial h_{1 m}}{\partial \tau}\right) \\
& +\left[b_{1}(\tau, x, 0)+i \omega_{m} b_{2}(\tau, x, 0)-2 i \omega_{m} \mu_{m}\right] \exp \left(i \omega_{m} t\right) \sin m x
\end{aligned}
$$

явньй вид (1.35)-(1.38) решения $h_{1 m}$ и формулы $(1.25),(1.26)$. В итоге убеждаемся, что

$$
\begin{aligned}
h_{2 m}= & D_{m}^{0}(x) \exp \left(i \omega_{m} t\right)+D_{m}^{+}(x) \exp \left[i\left(\omega_{m} t+2 \omega_{n} \tau\right)\right] \\
& +D_{m}^{-}(x) \exp \left[i\left(\omega_{m} t-2 \omega_{n} \tau\right)\right]
\end{aligned}
$$

где $D_{m}^{0}, D_{m}^{ \pm}-$решения краевых задач

$$
\begin{aligned}
-a^{2}\left(\frac{d^{2}}{d x^{2}} D_{m}^{0}+m^{2} D_{m}^{0}\right)=\psi_{m}^{0}(x), & D_{m}^{0}(0)=D_{m}^{0}(\pi)=0 \\
{\left[1-\left(\omega_{m} \pm 2 \omega_{n}\right)^{2}\right] D_{m}^{ \pm}-a^{2} \frac{d^{2}}{d x^{2}} D_{m}^{ \pm}=\psi_{m}^{ \pm}(x), } & D_{m}^{ \pm}(0)=D_{m}^{ \pm}(\pi)=0
\end{aligned}
$$

Здесь

$$
\begin{aligned}
\psi_{m}^{0}(x)= & i \omega_{m}\left(1-2 \mu_{m}\right) \sin m x+\widetilde{\psi}_{m}^{0}(x) \\
\widetilde{\psi}_{m}^{0}(x)= & \xi_{n}\left[2 a_{1}+i \omega_{m} a_{2}+2 \omega_{n}\left(\omega_{m}+\omega_{n}\right) a_{3}\right] C_{m}^{+}(x) \sin n x \\
& +\xi_{n}\left[2 a_{1}+i \omega_{m} a_{2}-2 \omega_{n}\left(\omega_{m}-\omega_{n}\right) a_{3}\right] C_{m}^{-}(x) \sin n x \\
& +\xi_{n}^{2}\left[2 a_{1} A(x)+\left(6 b_{1}+2 \omega_{n}^{2} b_{3}\right) \sin ^{2} n x\right] \sin m x \\
& +i \omega_{m} \xi_{n}^{2}\left[a_{2} A(x)+\left(2 b_{2}+6 \omega_{n}^{2} b_{4}\right) \sin ^{2} n x\right] \sin m x \\
\psi_{m}^{ \pm}(x)= & r_{1}^{ \pm} \cos \left[\left(\sigma_{m, 1}^{ \pm}+n\right) x\right]+r_{2}^{ \pm} \cos \left[\left(\sigma_{m, 1}^{ \pm}-n\right) x\right]+r_{3}^{ \pm} \sin \left[\left(\sigma_{m, 1}^{ \pm}+n\right) x\right] \\
& +r_{4}^{ \pm} \sin \left[\left(\sigma_{m}^{ \pm}-n\right) x\right]+r_{5}^{ \pm} \cos \left[\left(\sigma_{n}+m\right) x\right]+r_{6}^{ \pm} \cos \left[\left(\sigma_{n}-m\right) x\right] \\
& +r_{7}^{ \pm} \sin \left[\left(\sigma_{n}+m\right) x\right]+r_{8}^{ \pm} \sin \left[\left(\sigma_{n}-m\right) x\right]+r_{9}^{ \pm} \sin m x \\
& +r_{10}^{ \pm} \sin (m+2 n) x+r_{11}^{ \pm} \sin (m-2 n) x
\end{aligned}
$$

а явные формулы для коэффициентов $r_{j}^{ \pm}, j=1, \ldots, 11$, опустим, поскольку в дальнейшем они не потребуются.

Исследование первой краевой задачи (1.42) аналогично описанному выше анализу первой из задач (1.13): сначала из условия ее разрешимости находим неизвестную постоянную $\mu_{m}$, для которой в силу (1.43) получается равенство

$$
\mu_{m}=\frac{1}{2}-\frac{i}{\pi \omega_{m}} \int_{0}^{\pi} \widetilde{\psi}_{m}^{0}(x) \sin m x d x .
$$


Учитывая, далее, в (1.46) явњый вид (1.44) неоднородности $\widetilde{\psi}_{m}^{0}$, убеждаемся в существовании при $m \rightarrow \infty$ у последовательности $\mu_{m}$ конечного предела $\mu_{\infty}$.

После определения $\mu_{m}$ неоднородность (1.43) принимает вид

$$
\begin{aligned}
\psi_{m}^{0}(x)= & s_{1} \exp (x / a) \sin m x+s_{2} \exp (x / a) \cos m x+s_{3} \exp (-x / a) \sin m x \\
& +s_{4} \exp (-x / a) \cos m x+s_{5} \sin m x+s_{6} \sin (m+2 n) x+s_{7} \sin (m-2 n) x \\
& +s_{8} \cos \left[\left(\sigma_{m, 1}^{+}+n\right) x\right]+s_{9} \sin \left[\left(\sigma_{m, 1}^{+}+n\right) x\right]+s_{10} \cos \left[\left(\sigma_{m, 1}^{+}-n\right) x\right] \\
& +s_{11} \sin \left[\left(\sigma_{m, 1}^{+}-n\right) x\right]+s_{12} \cos \left[\left(\sigma_{m, 1}^{-}+n\right) x\right]+s_{13} \sin \left[\left(\sigma_{m, 1}^{-}+n\right) x\right] \\
& +s_{14} \cos \left[\left(\sigma_{m, 1}^{-}-n\right) x\right]+s_{15} \sin \left[\left(\sigma_{m, 1}^{-}-n\right) x\right]
\end{aligned}
$$

где (по той же причине, что и в случае (1.45)) явные формулы для коэффициентов $s_{j}, j=1, \ldots, 15$, опустим. А отсюда автоматически следует равенство

$$
D_{m}^{0}(x)=\widetilde{D}_{m}^{0}(x)-\widetilde{D}_{m}^{0}(0) \cos m x+\widetilde{s}_{5} x \cos m x
$$

в котором $\widetilde{s}_{5}=s_{5} /\left(2 m a^{2}\right)$, а $\widetilde{D}_{m}^{0}(x)$ получается из $\psi_{m}^{0}(x)-s_{5} \sin m x$ посредством замены $s_{j}, j \neq 5$, на $\widetilde{s}_{j}$, где

$$
\begin{aligned}
\widetilde{s}_{1} & =-\frac{s_{1}+2 a m s_{2}}{1+4 a^{2} m^{2}}, & \widetilde{s}_{2} & =\frac{2 a m s_{1}-s_{2}}{1+4 a^{2} m^{2}}, \\
\widetilde{s}_{3} & =\frac{2 a m s_{2}-s_{1}}{1+4 a^{2} m^{2}}, & \widetilde{s}_{4} & =-\frac{2 a m s_{1}+s_{2}}{1+4 a^{2} m^{2}}, \\
\widetilde{s}_{6} & =\frac{s_{6}}{\omega_{m+2 n}^{2}-\omega_{m}^{2}}, & \widetilde{s}_{7} & =\frac{s_{7}}{\omega_{m-2 n}^{2}-\omega_{m}^{2}}, \\
\widetilde{s}_{j} & =\frac{s_{j}}{a^{2}\left(\sigma_{m, 1}^{+}+n\right)^{2}-a^{2} m^{2}}, j=8,9 ; & \widetilde{s}_{j} & =\frac{s_{j}}{a^{2}\left(\sigma_{m, 1}^{+}-n\right)^{2}-a^{2} m^{2}}, j=10,11 ; \\
\widetilde{s}_{j} & =\frac{s_{j}}{a^{2}\left(\sigma_{m, 1}^{-}+n\right)^{2}-a^{2} m^{2}}, j=12,13 ; & \widetilde{s}_{j} & =\frac{a^{2}\left(\sigma_{m, 1}^{-}-n\right)^{2}-a^{2} m^{2}}{a^{2}}, j=14,15 .
\end{aligned}
$$

Обращаем внимание, что знаменатели в формулах для $\widetilde{s}_{j}, j=6, \ldots, 15$, отличны от нуля при любом $a>0$. Действительно, условия

$$
\omega_{m \pm 2 n}^{2}-\omega_{m}^{2} \neq 0
$$

эквивалентны очевидным неравенствам $4 a^{2} n(m \pm n) \neq 0$ (напомним, что $m \neq n$ ). Отличие же от нуля всех остальных знаменателей устанавливается однотипно. Проверим, например, выполнение условий

$$
a^{2} m^{2}-a^{2}\left(\sigma_{m, 1}^{-} \pm n\right)^{2} \neq 0
$$

С этой целью раскроем в них скобки и учтем явные формулы (1.33) для $\sigma_{m, 1}^{-}$. В итоге интересующие нас неравенства преобразуются к виду

$$
\pm 2 a n \sqrt{\left(\omega_{m}-\omega_{n}\right)^{2}-1} \neq 2 \omega_{m} \omega_{n}-2 \omega_{n}^{2}+1 .
$$

Возводя, далее, левую и правую части получившихся соотношений в квадрат, после некоторых преобразований приходим к условию

$$
4\left(\omega_{n}-\omega_{m}\right)^{2}+4 \omega_{n} \omega_{m}-3 \neq 0,
$$

справедливому в силу очевидного неравенства $4 \omega_{n} \omega_{m}-3>0$. 
Добавим еще, что после деления на $\omega_{m}$ все рассмотренные выше знаменатели стремятся при $m \rightarrow \infty$ к ненулевым конечным пределам. А так как при выполнении неравенств (1.34) модули коэффициентов $s_{j}, j=1, \ldots, 15$, при $m \rightarrow \infty$ растут не быстрее $\omega_{m}$, то отсюда вытекают аналогичные (1.40) оценки

$$
\left|\widetilde{s}_{j}\right| \leqslant N, \quad j=1, \ldots, 15 .
$$

Анализ оставшихся краевых задач (1.42) не вызывает затруднений, поскольку условия (1.34) гарантируют их однозначную разрешимость. Проводя соответствующие вычисления, в данном случае приходим к формулам

$$
D_{m}^{ \pm}(x)=D_{m, 0}^{ \pm}(x)-D_{m, 0}^{ \pm}(0) \theta_{*, m}^{ \pm, 2}(\pi-x)-D_{m, 0}^{ \pm}(\pi) \theta_{*, m}^{ \pm, 2}(x)
$$

Здесь для функций $D_{m, 0}^{ \pm}(x)$ справедливы аналогичные $(1.45)$ равенства, в которых коэффициенты $r_{j}^{ \pm}$следует заменить на $\widetilde{r}_{j}^{ \pm}$, где

$$
\begin{array}{ll}
\widetilde{r}_{j}^{ \pm}=r_{j}^{ \pm} /\left[1-\left(\omega_{m} \pm 2 \omega_{n}\right)^{2}+a^{2}\left(\sigma_{m, 1}^{ \pm}+n\right)^{2}\right], & j=1,3 ; \\
\widetilde{r}_{j}^{ \pm}=r_{j}^{ \pm} /\left[1-\left(\omega_{m} \pm 2 \omega_{n}\right)^{2}+a^{2}\left(\sigma_{m, 1}^{ \pm}-n\right)^{2}\right], & j=2,4 ; \\
\widetilde{r}_{j}^{ \pm}=r_{j}^{ \pm} /\left[1-\left(\omega_{m} \pm 2 \omega_{n}\right)^{2}+a^{2}\left(\sigma_{n}+m\right)^{2}\right], & j=5,7 ; \\
\widetilde{r}_{j}^{ \pm}=r_{j}^{ \pm} /\left[1-\left(\omega_{m} \pm 2 \omega_{n}\right)^{2}+a^{2}\left(\sigma_{n}-m\right)^{2}\right], & j=6,8 ; \\
\widetilde{r}_{9}^{ \pm}=r_{9}^{ \pm} /\left[\omega_{m}^{2}-\left(\omega_{m} \pm 2 \omega_{n}\right)^{2}\right], & \\
\widetilde{r}_{10}^{ \pm}=r_{10}^{ \pm} /\left[\omega_{m+2 n}^{2}-\left(\omega_{m} \pm 2 \omega_{n}\right)^{2}\right], & \\
\widetilde{r}_{11}^{ \pm}=r_{11}^{ \pm} /\left[\omega_{m-2 n}^{2}-\left(\omega_{m} \pm 2 \omega_{n}\right)^{2}\right] . &
\end{array}
$$

Отметим, что, как и выше, все знаменатели в коэффициентах $\widetilde{r}_{j}^{ \pm}$отличны от нуля. Убедимся, к примеру, в справедливости неравенств

$$
\begin{gathered}
\omega_{m}^{2}-\left(\omega_{m} \pm 2 \omega_{n}\right)^{2} \neq 0, \quad\left(\omega_{m}+2 \omega_{n}\right)^{2}-\omega_{m \pm 2 n}^{2} \neq 0 \\
\left(\omega_{m}-2 \omega_{n}\right)^{2}-\omega_{m \pm 2 n}^{2} \neq 0 .
\end{gathered}
$$

С этой целью, раскрьвая скобки, перепишем второе и третье из них в эквивалентной форме

$$
\left|1 \pm a^{2} m n\right| \neq \sqrt{1+a^{2} m^{2}} \sqrt{1+a^{2} n^{2}}
$$

А так как $m \neq n$, то в силу неравенства Буняковского-Коши левая часть (1.51) строго меньше правой. Что же касается первого неравенства (1.50), то оно эквивалентно очевидному неравенству $4 \omega_{n}\left(\omega_{n} \pm \omega_{m}\right) \neq 0$.

Отличие от нуля всех остальных знаменателей в $\widetilde{r}_{j}^{ \pm}$устанавливается подобно тому, как это сделано в случае (1.48). Поэтому соответствующие выкладки опустим. Отметим только, что здесь (по тем же причинам, что и выше) имеют место оценки

$$
\left|\widetilde{r}_{j}^{ \pm}\right| \leqslant N, \quad j=1, \ldots, 11
$$


Перед формулировкой основного результата введем в рассмотрение числа $R_{n, m}=\operatorname{Re} \mu_{m}(m \neq n), R_{n, \infty}=\operatorname{Re} \mu_{\infty}$, для которых из (1.44), (1.46) вытекают равенства:

$$
\begin{aligned}
R_{n, m}= & R_{n, m}^{0}-\frac{\xi_{n}^{2} \beta_{1}}{2 \omega_{n}}\left(1-\frac{1}{\omega_{2 n}^{2}}\right) \frac{4 m^{2} a^{2}}{1+4 m^{2} a^{2}} \Delta_{1}\left(\frac{\pi}{2 a}\right)+\frac{\xi_{n}^{2} a^{2}}{\pi \omega_{n}} \beta_{1} \\
& \times\left[\left(2+\frac{\omega_{n}}{\omega_{m}}\right) \sigma_{m, 1}^{+} \Delta_{n, m}^{+}\left(\pi \sigma_{m, 1}^{+}\right)+\left(2-\frac{\omega_{n}}{\omega_{m}}\right) \sigma_{m, 1}^{-} \Delta_{n, m}^{-}\left(\pi \sigma_{m, 1}^{-}\right)\right] \\
R_{n, \infty}= & R_{n, \infty}^{0}-\frac{\xi_{n}^{2} \beta_{1}}{2 \omega_{n}}\left(1-\frac{1}{\omega_{2 n}^{2}}\right) \Delta_{1}\left(\frac{\pi}{2 a}\right)
\end{aligned}
$$

где, напомним, постоянная $\beta_{1}$ определена в $(1.19), \Delta_{1}=\Delta_{1}(s)$ - функция из (1.17), а функции $\Delta_{n, m}^{ \pm}=\Delta_{n, m}^{ \pm}(s)$ и постоянные $R_{n, m}^{0}, R_{n, \infty}^{0}$ задаются формулами

$$
\begin{aligned}
\Delta_{n, m}^{ \pm}(s)= & \left(\frac{1}{\omega_{m+n}^{2}-\left(\omega_{m} \pm \omega_{n}\right)^{2}}-\frac{1}{\omega_{m-n}^{2}-\left(\omega_{m} \pm \omega_{n}\right)^{2}}\right)^{2} \frac{(-1)^{n+m}-\cos s}{\sin s} \\
2 R_{n, m}^{0}= & \frac{\xi_{n}^{2}}{2 \omega_{n}} \beta_{1}\left(2+\frac{\omega_{n}}{\omega_{m}}\right)\left[\frac{1}{\omega_{m+n}^{2}-\left(\omega_{m}+\omega_{n}\right)^{2}}+\frac{1}{\omega_{m-n}^{2}-\left(\omega_{m}+\omega_{n}\right)^{2}}\right] \\
& +\frac{\xi_{n}^{2}}{2 \omega_{n}} \beta_{1}\left(2-\frac{\omega_{n}}{\omega_{m}}\right)\left[\frac{1}{\omega_{m+n}^{2}-\left(\omega_{m}-\omega_{n}\right)^{2}}+\frac{1}{\omega_{m-n}^{2}-\left(\omega_{m}-\omega_{n}\right)^{2}}\right] \\
& +2 R_{n, \infty}^{0} \\
2 R_{n, \infty}^{0}=1 & +\left(\beta_{1} / \omega_{n}+b_{2}+3 \omega_{n}^{2} b_{4}\right) \xi_{n}^{2} .
\end{aligned}
$$

ТЕОРЕМА 1.1. Пусть при некотором натуральном $п$ справедливы неравенства (1.11), (1.20), (1.22), (1.23), (1.34), отличны от нуля числа (1.53), (1.54) и количество положительных среди них равно $m_{0}$. Тогда найдется такое $\varepsilon_{0}>0$, что при всех $0<\varepsilon \leqslant \varepsilon_{0}$ краевая задача (1.1) имеет иикл с асимптотикой (1.3), (1.4), (1.21), әкспоненциально орбитально устойчивый при $m_{0}=0$ и дихотомичный при $m_{0}>0$ с размерностью неустойчивого многообразия $2 m_{0}+1$.

Из формул (1.17)-(1.19), (1.53)-(1.57) следует, что при подходящем выборе коэффициентов $a_{1}, a_{2}, a_{3}$ и $b_{2}, b_{4}$ разложения (1.5) краевая задача (1.1) может иметь любое конечное число устойчивых циклов, а также циклы с бесконечномерными неустойчивыми многообразиями (при $R_{n, \infty}>0$ ). Что же касается неравенств $(1.11),(1.22),(1.23),(1.34)$, то они представляют собой ограничения на параметр $a$ и заведомо вьполняются при некоторой общности положения.

1.2. Доказательство теоремы 1.1. Для начала обоснуем описанный выше алгоритм асимптотического вычисления характеристических показателей краевой задачи (1.24). Введем новые переменные $h_{1}=h_{t}, h_{2}=B h$, где $B$ - арифметический квадратный корень из оператора $-a^{2} d^{2} / d x^{2}+I$ с граничными условиями Дирихле, и перейдем обычным образом от уравнения (1.24) к системе первого порядка в $\stackrel{\circ}{W} \frac{1}{2} \times \stackrel{\circ}{W} \underset{2}{1}$. Выполним затем в получившейся системе замену, исходя из 
формулы

$$
\begin{aligned}
h= & \left(V_{0}+\sqrt{\varepsilon} V_{1}+\varepsilon V_{2}\right) v+\sum_{\substack{m=1 \\
m \neq n}}^{\infty}\left\{\omega_{m}^{-2}\left[\exp \left(i \omega_{m} t\right) \sin m x+\sqrt{\varepsilon} h_{1 m}+\varepsilon h_{2 m}\right] \eta_{m}\right. \\
& \left.+\omega_{m}^{-2}\left[\exp \left(-i \omega_{m} t\right) \sin m x+\sqrt{\varepsilon} \bar{h}_{1 m}+\varepsilon \bar{h}_{2 m}\right] \bar{\eta}_{m}\right\},
\end{aligned}
$$

где $v=\operatorname{colon}\left(\eta_{n}, \bar{\eta}_{n}\right), \eta=\operatorname{colon}\left(\eta_{1}, \bar{\eta}_{1}, \eta_{2}, \bar{\eta}_{2}, \ldots\right) \in l_{2}$. Последнее означает, что при дифференцировании (1.58) по $t$ следует считать

$$
\dot{v}=\varepsilon D v ; \quad \dot{\eta}_{m}=\varepsilon \mu_{m} \eta_{m}, \quad m \neq n,
$$

где $D$ - матрица (1.28). Заметим, далее, что из способа построения и свойств входящих в (1.58) функций (см. оценки $(1.40),(1.49),(1.52))$ следует, что формула (1.58) индуцирует ограниченньй (равномерно по $t, \varepsilon$ ) оператор, действующий из $l_{2}$ в $\stackrel{\circ}{W} \underset{2}{1} \times \stackrel{\circ}{W} \underset{2}{1}$, а результатом применения указанной замены к уравнению $(1.24)$ служит система в $l_{2}$ :

$$
\dot{\eta}=\varepsilon \Lambda_{0} \eta+\varepsilon^{3 / 2} \Lambda_{1}(t, \varepsilon) \eta
$$

Здесь

$$
\Lambda_{0}=\operatorname{diag}\left\{\mu_{1}, \bar{\mu}_{1}, \ldots, \mu_{n-1}, \bar{\mu}_{n-1}, D, \mu_{n+1}, \bar{\mu}_{n+1}, \ldots\right\},
$$

а линейный оператор $\Lambda_{1}$ ограничен равномерно по $t \in \mathbb{R}$ и $\varepsilon$ (по поводу построения $\Lambda_{1}$ см. соответствуюшие места в [10], [15]).

Несложный анализ системы (1.59) показывает, что ее свойства устойчивости совпадают с теми, о которых говорится в теореме: реальные части всех ее характеристических показателей (за исключением одного, имеющего порядок $\varepsilon^{3 / 2}$ ) равномерно по $m \geqslant 1$ с точностью до $\varepsilon^{3 / 2}$ совпадают с числами $\varepsilon \operatorname{Re} \mu_{m}(m \neq n)$ и - $\varepsilon$.

Завершающий этап - доказательство сушествования у краевой задачи (1.1) периодического решения с требуемой асимптотикой-проводится по стандартной схеме из [12]. Выполним в (1.1) замену времени $\tau=\left(1+\varepsilon \alpha_{n}+\varepsilon^{2} \delta\right) t$, где $\delta=\delta(\varepsilon)-$ подлежащая определению ограниченная функция $\varepsilon$, и положим

$$
u=\varepsilon^{1 / 2} u_{1}(\tau, x)+\varepsilon u_{2}(\tau, x)+\varepsilon^{3 / 2} u_{3}(\tau, x)+\varepsilon^{2} u_{4}(\tau, x)+\varepsilon^{3 / 2} h,
$$

где функции $u_{k}, k=1, \ldots, 4$, взяты из (1.3). В результате приходим к уравнению

$$
\Pi(\varepsilon, \delta) h=\varepsilon F\left(\tau, x, \varepsilon, \delta, h, h_{\tau}\right),
$$

в котором

$$
\begin{aligned}
\Pi(\varepsilon, \delta) h= & \left(1+\varepsilon \alpha_{n}+\varepsilon^{2} \delta\right)^{2} h_{\tau \tau}-a^{2} h_{x x}+h \\
& -\varepsilon^{1 / 2}\left[a_{1}(\tau, x) h+a_{2}(\tau, x)\left(1+\varepsilon \alpha_{n}+\varepsilon^{2} \delta\right) h_{\tau}\right] \\
& -\varepsilon\left[b_{1}(\tau, x, \varepsilon) h+b_{2}(\tau, x, \varepsilon)\left(1+\varepsilon \alpha_{n}+\varepsilon^{2} \delta\right) h_{\tau}\right],
\end{aligned}
$$

а функция $F(\tau, x, \varepsilon, \delta, u, v)$ является гладкой по совокупности переменнњх, $2 \pi / \omega_{n^{-}}$ периодической по $\tau$ и такова, что

$$
\begin{gathered}
\sup _{\tau}\|F(\tau, x, \varepsilon, \delta, 0,0)\|_{\stackrel{\circ}{2}_{2}^{1}} \leqslant N_{1}, \\
\sup _{\tau}\left\|F\left(\tau, x, \varepsilon, \delta, u_{1}, v_{1}\right)-F\left(\tau, x, \varepsilon, \delta, u_{2}, v_{2}\right)\right\|_{\stackrel{\circ}{2}_{2}^{1}} \leqslant N_{2} \varepsilon^{1 / 2}\left(\left|u_{1}-u_{2}\right|+\left|v_{1}-v_{2}\right|\right),
\end{gathered}
$$

где $N_{2}=N_{2}(R), R=\max \left\{\left|u_{j}\right|,\left|v_{j}\right|, j=1,2\right\}$. 
Из наших построений следует, что уравнение

$$
\Pi(\varepsilon, \delta) h=0
$$

имеет приближенное (с точностью до $\varepsilon^{2}$ по невязке) периодическое решение

$$
h_{0}(\tau, x, \varepsilon)=\frac{\partial}{\partial \tau}\left[u_{1}(\tau, x)+\varepsilon^{1 / 2} u_{2}(\tau, x)+\varepsilon u_{3}(\tau, x)+\varepsilon^{3 / 2} u_{4}(\tau, x)\right] \text {. }
$$

Добавляя в левую часть этого уравнения слагаемое (имеющее порядок $\varepsilon^{2}$ )

$$
-\Delta(\tau, x, \varepsilon, \delta) l(h) / l\left(h_{0}\right)
$$

где

$$
\Delta=\Pi(\varepsilon, \delta) h_{0}, \quad l(h)=\int_{0}^{\pi}\left[h \omega_{n} \sin \omega_{n} \tau+\frac{\partial h}{\partial \tau} \cos \omega_{n} \tau\right] \sin n x d x
$$

можно сделать его точным, что и полагаем ниже. Естественно, что добавку (1.66) нужно учесть и в правой части (1.61), общие свойства которой от этого не изменятся.

Рассмотрим оператор П $(\varepsilon, \delta)$ в банаховом пространстве $H$, состоящем из таких $2 \pi / \omega_{n}$-периодических по $\tau$ функций $h(\tau, x)$, что сама $h(\tau, x)$ непрерывна по $\tau$ в метрике $\stackrel{\circ}{W} \underset{2}{2}, h_{\tau}$ непрерьвна в метрике $\stackrel{\circ}{W} \underset{2}{1}$, а $h_{\tau \tau}$ - в метрике $L_{2}$. Норму в $H$ определим формулой

$$
\|h\|_{H}=\max _{\tau}\left\{\|h\|_{\stackrel{\circ}{2}_{2}^{2}}+\left\|h_{\tau}\right\|_{\stackrel{\circ}{W}_{2}^{1}}+\left\|h_{\tau \tau}\right\|{ }_{L_{2}}\right\} .
$$

Введем еще пространство $H_{0}$, состоящее из непрерывных $2 \pi / \omega_{n}$-периодических по $\tau$ функций $h(\tau, x)$ со значениями в $\stackrel{\circ}{W} \underset{2}{1}$ и нормой

$$
\|h\|_{H_{0}}=\max _{\tau}\|h(\tau, x)\|_{W_{2}^{1}}^{\stackrel{\circ}{1}}
$$

Как известно, необходимым и достаточным условием разрешимости в $H$ уравнения

$$
\Pi(\varepsilon, \delta) h=G(\tau, x), \quad G \in H_{0}
$$

является равенство

$$
\frac{\omega_{n}}{2 \pi} \int_{0}^{2 \pi / \omega_{n}} \int_{0}^{\pi} G(\tau, x) g(\tau, x, \varepsilon, \delta) d x d \tau=0
$$

где $g(\tau, x, \varepsilon, \delta)$ - периодическое решение сопряженного с (1.65) уравнения, удовлетворяющее условию нормировки

$$
\frac{\omega_{n}}{2 \pi} \int_{0}^{2 \pi / \omega_{n}} \int_{0}^{\pi} g(\tau, x, \varepsilon, \delta) \frac{\partial}{\partial \tau} h_{0}(\tau, x, \varepsilon) d x d \tau=1
$$

Решение $h_{G}$ уравнения (1.67) при этом единственно, если $\partial h_{G} / \partial \tau$ ортогонально в среднем функции $g$. Кроме этого, имеет место неравенство

$$
\left\|h_{G}\right\|_{H} \leqslant \frac{N_{3}}{\varepsilon}\|G\|_{H_{0}}
$$


вытекающее из структуры замены (1.58) и вида системы (1.59), к которой преобразуется уравнение (1.24) с помошью указанной замены.

Рассматривая правую часть уравнения (1.61) как неоднородность $G$ и подправляя ее соответствующим образом (вычитая из $G$ функцию $\partial h_{0} / \partial \tau$, умноженную на левую часть (1.68)), переходим к интегральному уравнению, обращая оператор П. Неравенства (1.63), (1.64), (1.70) позволяют применить к нему в $H$ принцип сжимающих отображений и определить функцию $h(\tau, x, \varepsilon, \delta):\|h\|_{H} \leqslant N_{4}$. Подставляя затем ее в равенство (1.68) (т.е. зануляя сделанную ранее поправку к правой части уравнения (1.61)), для отыскания $\delta$ получаем уравнение

$$
\delta=p(\varepsilon)+\varepsilon^{1 / 2} \Omega(\varepsilon, \delta),
$$

где функция $p(\varepsilon)$ ограничена.

Можно показать (соответствующий анализ для уравнения $(*)$ с граничными условиями Неймана проделан, например, в [16]), что функции $\Omega(\varepsilon, \delta)$ и $\Omega_{\delta}^{\prime}(\varepsilon, \delta)$ ограничены равномерно по $\varepsilon$ на любом компактном множестве изменения переменной $\delta$. Поэтому, применяя к уравнению (1.71) теорему о неявной функции, однозначно определяем интересуюшую нас поправку к частоте $\delta=\delta(\varepsilon)$. Теорема 1.1 доказана.

Отметим, что наиболее простая ситуация реализуется в рамках краевой задачи (1.1) при условиях

$$
a_{1}=a_{2}=a_{3}=0
$$

Действительно, в этом случае отпадает надобность в неравенствах (1.11), (1.22), так как заведомо $u_{2} \equiv 0$ и отсутствует первое слагаемое в формуле (1.15). Далее, при проведении алгоритма исследования устойчивости нет необходимости требовать выполнения условий (1.34), так как здесь $h_{1 m} \equiv 0$, а коэффициенты $D_{m}^{0}(x)$ и $D_{m}^{ \pm}(x)$ из (1.41) являются линейными комбинациями только функций $\sin (m \pm 2 n) x$ и $\sin m x, \sin (m \pm 2 n) x$ соответственно. И наконец, в данном случае лишними оказываются и неравенства (1.23), обеспечивающие возможность определения функции $u_{4}$ из (1.3). Связано это с тем, что при условиях (1.72) в доказательстве теоремы 1.1 можно вместо (1.60) положить

$$
u=\varepsilon^{1 / 2} u_{1}(\tau, x)+\varepsilon u_{2}(\tau, x)+\varepsilon^{3 / 2} u_{3}(\tau, x)+\varepsilon h .
$$

В результате получим аналогичное (1.61) уравнение, правая часть $F$ которого обладает прежними свойствами (1.63), (1.64). Для сравнения заметим, что если осуществить замену равенства (1.60) на (1.73) в общем случае (т.е. при отказе от требований $(1.72))$, то исчезнет множитель $\varepsilon^{1 / 2}$ в правой части неравенства (1.64). А это, в свою очередь, сделает невозможньг последующее применение к получающемуся из (1.61) интегральному уравнению принципа сжимающих отображений.

Итак, в обшем случае для доказательства сушествования у краевой задачи (1.1) точного периодического решения с асимптотикой $(1.3),(1.4)$ приближенное периодическое решение необходимо знать с точностью до слагаемых порядка $\varepsilon^{2}$ включительно. При этом, однако, от первой групшы ограничений (1.23) все же можно отказаться. В самом деле, пусть $2 \omega_{n}=\omega_{2 k_{0}}$ при некотором натуральном $k_{0}$. Тогда вырожденной окажется краевая задача для коэффициента функции $u_{4}(\tau, x)$ при 
гармонике $\exp \left(2 i \omega_{n} \tau\right)$. Разрешимости ее можно добиться, если учесть в $u_{2}(\tau, x)$ слагаемое

$$
\left[\eta \exp \left(2 i \omega_{n} \tau\right)+\bar{\eta} \exp \left(-2 i \omega_{n} \tau\right)\right] \sin 2 k_{0} x
$$

где $\eta$ - произвольная комплексная постоянная. Подчеркнем, что в силу очевидного равенства

$$
\int_{0}^{\pi} \sin 2 k_{0} x \sin ^{2} n x d x=0
$$

слагаемое (1.74) не даст вклада в условие разрешимости первой из задач (1.13), а постоянная $\eta$ определится из условия разрешимости краевой задачи для интересуюшего нас коэффициента функции $u_{4}$. Несложный подсчет показывает, что для $\eta$ выйдет линейное неоднородное уравнение вида $\mu_{2 k_{0}} \eta=\varphi_{0}$, однозначно разрешимое в силу предполагаемого в условиях теоремы 1.1 неравенства $\operatorname{Re} \mu_{2 k_{0}} \neq 0$.

Используя описанный выше прием, в принципе, можно избавиться и от второй группы ограничений (1.23). Однако в отличие от предыдушего случая, в котором при последуюшей реализации алгоритма исследования устойчивости не возникает дополнительных условий, в данной ситуации (т.е. при выполнении равенства $4 \omega_{n}=\omega_{k_{0}}$ при некотором натуральном $k_{0}$ ) необходимо еще убедиться в справедливости условий (1.34) при $p=4$.

Отдельно остановимся на роли второй групшы неравенств (1.34). Пусть, например, $\omega_{n}=a k_{0}$ при некотором натуральном $k_{0}$. Тогда при выполнении всех прочих ограничений формально правая часть (1.58) может быть построена. Но поскольку здесь $\left|\sin \left(\pi \sigma_{m, 1}^{ \pm}\right)\right| \rightarrow 0$ при $m \rightarrow \infty$, то возникает так называемый эффект малых знаменателей: не все входящие в $h_{1 m}, h_{2 m}$ коэффициенты ограничены равномерно по $m$ и вследствие этого замена (1.58) становится некорректной (не порождает ограниченного оператора, действуюшего из $l_{2}$ в $\left.\stackrel{\circ}{W} \underset{2}{1} \times \stackrel{\circ}{W} \underset{2}{1}\right)$.

В качестве конкретного примера, иллюстрирующего содержательность теоремы 1.1, рассмотрим краевую задачу

$$
u_{t t}-\varepsilon u_{t}+u=a^{2} u_{x x}-u^{2} u_{t},\left.\quad u\right|_{x=0}=\left.u\right|_{x=\pi}=0
$$

В данном случае условие (1.20) выполняется при любом натуральном $n$, так как $d_{n}=-3 \omega_{n} / 4$. Надобность же во всех остальных ограничениях отпадает, поскольку здесь справедливы равенства (1.72) и кроме этого

$$
u_{2}=u_{4}=0, \quad \xi_{n}=2 / \sqrt{3}, \quad \alpha_{n}=0, \quad \mu_{m}=\mu_{\infty}=-1 / 6
$$

Таким образом, вместо теоремы 1.1 получаем следующее утверждение.

ТЕОРема 1.2. Для любого натурального $n_{0}$ u для любъх $a_{* *}>a_{*}>0$ найдется такое $\varepsilon_{0}>0$, что при $0<\varepsilon \leqslant \varepsilon_{0}, a_{*} \leqslant a \leqslant a_{* *}$ краевая задача (1.75) имеет әкспоненциально орбитально устойчивые периодические решения с асимптотикой (1.3), (1.4) при $n=1,2, \ldots, n_{0}$.

Будем говорить, что в некоторой системе наблюдается явление буферности, если при подходящем выборе параметров можно гарантировать сушествование в ней любого наперед заданного конечного числа устойчивых циклов. Из теоремы 1.2 следует, что указанное явление имеет место в рамках краевой задачи (1.75). 
1.3. Случай граничных условий Неймана. В этом пункте для уравнения $(*)$ рассмотрим краевую задачу

$$
u_{t t}-\varepsilon u_{t}+u=a^{2} u_{x x}+f\left(u, u_{t}\right),\left.\quad u_{x}\right|_{x=0}=\left.u_{x}\right|_{x=\pi}=0
$$

и для построения ее периодических решений применим изложенные выше алгоритмы. А именно, фиксируем произвольно натуральное $n$ и подставим в (1.76) ряд (1.3), в котором теперь

$$
u_{1}=\left[\exp \left(i \omega_{n} \tau\right)+\exp \left(-i \omega_{n} \tau\right)\right] \xi_{n} \cos n x
$$

а частоты $\omega_{n}$ задаются прежними равенствами (см. (1.2)). Действуя затем описанньм в п. 1.1 способом, для нахождения $u_{k}, k=2,3,4$, получим аналогичную (1.6) рекуррентную последовательность линейных неоднородных краевых задач

$$
\left(\frac{\partial^{2}}{\partial \tau^{2}}-a^{2} \frac{\partial^{2}}{\partial x^{2}}\right) u_{k}+u_{k}=g_{k}(\tau, x),\left.\quad \frac{\partial u_{k}}{\partial x}\right|_{x=0, x=\pi}=0
$$

Анализ задачи (1.77) при $k=2$ приводит к выводу, что для $u_{2}$ справедливо прежнее представление (1.7). Однако теперь коэффициенты в нем задаются равенствами, получающимися из (1.9) при отбрасывании слагаемых, содержащих $\theta_{*}(x), \theta_{* *}(x)$, и при замене $\left(-1 / \omega_{2 n}^{2}\right) \cos 2 n x$ на $\left(1 / \omega_{2 n}^{2}\right) \cos 2 n x,(1 / 3) \cos 2 n x$ на $(-1 / 3) \cos 2 n x$. Главное же отличие от предыдушего случая состоит в том, что здесь не возникает никаких дополнительных ограничений вида (1.11).

При $k=3$, учитывая уже найденное решение $u_{2}$, убеждаемся, что правая часть в (1.77) - линейная комбинация функций

$$
\exp \left( \pm i m \omega_{n} \tau\right) \cos p n x, \quad m, p=1,3
$$

Поэтому для разрешимости задачи (1.77) при $k=3$ в классе тригонометрических полиномов переменной $\omega_{n} \tau$ необходимо и достаточно отсутствие в ее правой части слагаемых, пропорциональных $\exp \left( \pm i \omega_{n} \tau\right) \cos n x$. Приравнивая их к нулю, для определения неизвестных постоянных $\xi_{n}, \alpha_{n}$ приходим к уравнению $(1.16)$, в котором теперь ляпуновская величина $d_{n}$ задается равенством (1.18).

Предположим, что, как и выше, выполняется условие (1.20). Тогда из (1.16) находим постоянные $\xi_{n}, \alpha_{n}$, для которых справедливы прежние формулы (1.21), а затем определяем и саму функцию $u_{3}$ в виде линейной комбинации гармоник (1.78) при $m=1, p=3$ и $m=3, p=1,3$. И наконец, в случае $k=4$ разрешимость краевой задачи (1.77) в требуемом классе функций гарантирует условие

$$
a^{2} \neq(2 n)^{-2}
$$

возникающее при нахождении коэффициентов функции $u_{4}$ при гармониках $\exp \left( \pm 2 i \omega_{n} \tau\right) \cos 4 n x$. 
Следующий этап - применение алгоритмов исследования устойчивости из п. 1.1 к краевой задаче

$$
\begin{gathered}
h_{t t}-a^{2} h_{x x}+h=\varepsilon^{1 / 2}\left[a_{1}(\tau, x) h+a_{2}(\tau, x) h_{t}\right]+\varepsilon\left[b_{1}(\tau, x, \varepsilon) h+b_{2}(\tau, x, \varepsilon) h_{t}\right], \\
\left.h_{x}\right|_{x=0}=\left.h_{x}\right|_{x=\pi}=0,
\end{gathered}
$$

для коэффициентов которой по-прежнему справедливы равенства (1.25), (1.26).

Для расчета характеристических показателей краевой задачи (1.80) на частоте $\omega_{n}$ подставим в нее формулу (1.27), в которой теперь $w_{0}=\exp \left(i \omega_{n} \tau\right) \cos n x$, а матрица $D$ имеет прежнюю структуру (1.28). В результате снова приходим к равенствам (1.29).

При $m \neq n, m \geqslant 0$, подставляя в (1.80) выражение (1.30), в котором $\sin m x$ заменен на $\cos m x$, получаем краевые задачи вида (1.31), в которых, естественно, граничные условия Дирихле следует заменить на условия Неймана. Анализ первой из них достаточно прост, так как ее неоднородность $f_{1}-$ линейная комбинация гармоник

$$
\exp \left[i\left(\omega_{m} t+\omega_{n} \tau\right)\right] \cos (m \pm n) x, \quad \exp \left[i\left(\omega_{m} t-\omega_{n} \tau\right)\right] \cos (m \pm n) x
$$

Поэтому для нахождения $h_{1 m}$ в виде тригонометрического полинома той же структуры необходимо и достаточно выполнение неравенств (1.39), справедливость которых при всех $a>0$ уже установлена в п. 1.1.

Для нахождения $h_{2 m}$ сначала, приравнивая к нулю коэффициент при гармонике $\exp \left(i \omega_{m} t\right) \cos m x$ в $f_{2}$, определяем комплексную величину $\mu_{m}$, которая, как и в п. 1.1, имеет при $m \rightarrow \infty$ конечньй предел $\mu_{\infty}$. Явную формулу для $\mu_{m}$ опустим, так как в дальнейшем она не потребуется. Отметим лишш, что здесь для $R_{n, m}=\operatorname{Re} \mu_{m}, R_{n, \infty}=\operatorname{Re} \mu_{\infty}$ имеют место равенства (1.56), (1.57).

Завершая описание алгоритма, добавим, что поскольку после нахождения $\mu_{m}$ неоднородность $f_{2}$ представляет собой линейную комбинацию гармоник

$$
\begin{gathered}
\exp \left(i \omega_{m} t\right) \cos (m \pm 2 n) x, \quad \exp \left[i\left(\omega_{m} t+2 \omega_{n} \tau\right)\right] \cos (m \pm 2 n) x \\
\exp \left[i\left(\omega_{m} t-2 \omega_{n} \tau\right)\right] \cos (m \pm 2 n) x, \quad \exp \left[i\left(\omega_{m} t \pm 2 \omega_{n} \tau\right)\right] \cos m x
\end{gathered}
$$

то и $h_{2 m}$ уместно искать в том же виде. Однако для того чтобы указанное было возможным, необходимо и достаточно выполнение неравенств (1.47), (1.50). Справедливость же последних при всех $a>0$ установлена нами в п. 1.1. Добавим еще, что в данном случае автоматически отсутствуют малые знаменатели, т.е. все входящие в $h_{1 m}, h_{2 m}$ коэффицциенты при гармониках (1.81) и (1.82) соответственно допускают равномерные по $m$ оценки вида (1.40). А это, в свою очередь, позволяет при обосновании приведенных алгоритмов сконструировать корректную бесконечномерную замену переменных вида (1.58).

Суммируя все сказанное, приходим к выводу, что для краевой задачи (1.76) аналог теоремы 1.1 имеет место при минимальных ограничениях: для рассматриваемого номера $n$ следует требовать выполнения неравенств $(1.20),(1.79)$ и отличия от нуля чисел (1.56), (1.57). 
В заключение дадим сравнительный анализ динамики по параметру $а$ периодических решений краевых задач (1.1) и (1.76). С этой целью введем в рассмотрение комплексную ляпуновскую величину

$$
d=\frac{1}{2}\left[a_{2}\left(a_{1}+a_{3}\right)+b_{2}+3 b_{4}\right]-\frac{i}{6}\left[10 a_{1}\left(a_{1}+a_{3}\right)+4 a_{3}^{2}+a_{2}^{2}+9 b_{1}+3 b_{3}\right]
$$

обыкновенного уравнения, получающегося из $(*)$ при $\varepsilon=a=0$, и предположим, что

$$
\operatorname{Re} d<0 \text {. }
$$

Обратимся затем к краевой задаче (1.76) и будем рассматривать соответствуюшие ей величины $d_{n}, \xi_{n}, R_{n, m}, R_{n, \infty}$ как функции $d_{n}(a), \xi_{n}(a), R_{n, m}(a), R_{n, \infty}(a)$ параметра $a$ (все остальные параметры предполагаем фиксированными). Анализируя формулы (1.18), (1.56), (1.57), нетрудно увидеть, что

$$
d_{n}(0)=\frac{3}{2} d, \quad \xi_{n}(0)=\sqrt{-2 /(3 \operatorname{Re} d)}, \quad R_{n, \infty}(0)=-1 / 6
$$

и равномерно по $m \geqslant 0$

$$
\lim R_{n, m}(a)=-1 / 6, \quad a \rightarrow 0
$$

А это означает, что при условии (1.84) и при согласованном уменьшении параметров $a, \varepsilon$ в краевой задаче (1.76) происходит неограниченное увеличение числа устойчивых циклов, т.е. реализуется феномен буферности (см. определение, данное после теоремы 1.2).

В случае краевой задачи (1.1) все перечисленные факты заведомо справедливы в простейшей ситуации, когда выполняются равенства (1.72). Однако при некоторой общности положения, а именно при $\beta_{1} \neq 0$, функция $\operatorname{Re} d_{n}(a)$, определяющаяся теперь посредством формул (1.17)-(1.19), имеет в точках $a_{k}^{*}, k \geqslant n$ (см. (1.11)), полюсы первого порядка, сгущающиеся к точке $a=0$. А отсюда вытекает, что при $a \rightarrow 0$ она бесконечно много раз меняет знак. Поэтому найдутся такие сколь угодно близкие к нулю значения параметра $a$, при которых $\operatorname{Re} d_{n}(a)>0$, и, следовательно, у краевой задачи (1.1) не сушествует периодического решения (1.3), (1.4) с фиксированным номером $n$.

$\mathrm{C}$ другой стороны, заведомо найдутся и такие последовательности $a_{s} \rightarrow 0$, $s \rightarrow \infty$, значений $a$, при которых функции $d_{n}(a), \xi_{n}(a), R_{n, \infty}(a)$ стремятся к пределам из (1.85), а также сохраняются предельные равенства (1.86). Тем самњм, при выполнении условия (1.84) и при $\beta_{1} \neq 0$ для каждого периодического решения $(1.3),(1.4)$ задачи (1.1) наблюдается так называемый әффект мериания: сколь угодно малые значения параметра $a$, при которых оно устойчиво, перемежаются со значениями $a$, при которых данного решения не сушествует.

Итак, установлено, что динамика по параметру $а$ периодических решений краевой задачи (1.1) существенно зависит от наличия или отсутствия в разложении (1.5) квадратичных слагаемых, в то время как в случае краевой задачи (1.76) ничего подобного не наблюдается. В чисто аналитическом плане это объясняется 
тем, что система функций $\cos n x, n \geqslant 0$, обладает свойством замкнутости относительно операции умножения, которое выражается равенством

$$
\cos n x \cos m x=\frac{1}{2}[\cos (n+m) x+\cos (n-m) x]
$$

а у системы $\sin x, n \geqslant 1$, такое свойство отсутствует. Однако данное тривиальное обстоятельство приводит в случае краевой задачи (1.1) при $\beta_{1} \neq 0$ к весьма нетривиальньм последствиям. А именно, любое ее периодическое решение $(1.3),(1.4)$ с фиксированным номером $n$ при уменьшении параметра $a$ и при прохождении его через критические значения, соответствующие нарушению условий (1.11), (1.22), (1.34), претерпевает бесконечную последовательность бифуркаций. Добавим, наконец, что если нарушение условий $(1.11),(1.22)$ влияет на сам факт существования цикла с требуемой асимптотикой, то "материализация" комбинационных резонансов приводит к менее существенньм последствиям. Например, как следует из формул (1.53), (1.55), при прохождении параметра $a$ через критическое значение $a_{*}$, определяюшееся из условия $\omega_{k}=\omega_{n}+\omega_{m}$ при некоторых $k$ и $m$, может наблюдаться лишш смена устойчивости цикла (1.3), (1.4). Связано это с тем, что в случае нечетного $n+m+k$ функция $R_{n, m}(a)$ имеет в точке $a=a_{*}$ полюс первого порядка.

\section{§2. Метод квазинормальных форм и границы его применимости}

2.1. Общий случай. Для прояснения существа дела описание интересующего нас метода дадим сначала для некоторого обшего класса гиперболических систем. В связи с этим на отрезке $0 \leqslant x \leqslant \pi$ рассмотрим краевую задачу

$$
\begin{gathered}
u_{t t}+\left(A_{0}+\varepsilon A_{1}\right) u_{t}+\left(B_{0}+\varepsilon B_{1}\right) u=\varepsilon D u_{x x}+f\left(u, u_{t}\right), \\
\left.u\right|_{x=0}=\left.u\right|_{x=\pi}=0,
\end{gathered}
$$

где $u \in \mathbb{R}^{m}, m \geqslant 2 ; 0<\varepsilon \ll 1 ; A_{j}, B_{j}, j=0,1$, и $D$ - постоянные матрицы, причем $D^{*}=D, D>0$; тейлоровское разложение в нуле вектор-функции $f(u, v) \in$ $C^{\infty}$ начинается с квадратичных слагаемых. В качестве фазового пространства краевой задачи (2.1), как и в случае (1.1), возьмем

$$
\left(u, u_{t}\right) \in \stackrel{\circ}{W}=2\left([0, \pi] ; \mathbb{R}^{m}\right) \times \stackrel{\circ}{W_{2}^{1}}\left([0, \pi] ; \mathbb{R}^{m}\right) .
$$

Сформулируем условия, при которых будет изучаться проблема сушествования и устойчивости периодических по $t$ решений краевой задачи $(2.1)$, бифурцирующих из ее нулевого состояния равновесия при $\varepsilon>0$. Первое ограничение касается автоколебательных свойств уравнения из (2.1) при $\varepsilon=0$.

УСловИЕ 2.1. Предполагаем, что квадратичный пучок матрии, $\lambda^{2} I+\lambda A_{0}+$ $B_{0}$, где $I$ - единичная матрица, имеет простую пару чисто мнимых собственных значений $\pm i$, т.е. существуют такие векторы а и $b$, что

$$
\begin{aligned}
\left(-I+i A_{0}+B_{0}\right) a & =0, & & \left(-I-i A_{0}^{*}+B_{0}^{*}\right) b=0, \\
\left(2 i a+A_{0} a, b\right) & =1, & & \left(2 i a+A_{0} a, \bar{b}\right)=0,
\end{aligned}
$$

где $(*, *)$ - обичное скалярное произведение в комплексификачии $\mathbb{R}^{m}$. Остальные жсе собственные значения рассматриваемого пучка матрии считаем лежашими в полуплоскости $\operatorname{Re} \lambda<0$.

Следуюшее ограничение обеспечивает нужное согласование “обыкновенной” и “диффузионной” частей в (2.1). 
УСловИЕ 2.2. Считаем, что при всех $z>0$ собственные значения квадратичного пучка матрии,

$$
\lambda^{2} I+\lambda A_{0}+B_{0}+z D
$$

лежат в полуплоскости $\operatorname{Re} \lambda<0$, а при малых z те собственные значения $\lambda(z), \bar{\lambda}(z)$ пучка (2.3), для которых $\lambda(0)=i$ (их существование вытекает из условия 2.1), отходят от мнимой оси общим образом, т.е. выполняется неравенство

$$
-\left.\operatorname{Re} \lambda_{z}^{\prime}\right|_{z=0}=\operatorname{Re}(D a, b)>0
$$

Перед формулировкой очередного ограничения предположим для простоты, что все собственные значения $\lambda_{k}>0, k=1, \ldots, m$, матрицы $D$ различны, и обозначим через $e_{k}, k=1, \ldots, m$, соответствующую им ортонормированную систему собственных векторов.

УСЛОвИЕ 2.3. Предположим, что

$$
\left(A_{0} e_{k}, e_{k}\right)>0, \quad k=1, \ldots, m
$$

Остановимся на связи условий 2.2 и 2.3. Заметим, что собственные значения $\lambda_{k}(z), \bar{\lambda}_{k}(z), k=1, \ldots, m$, пучка (2.3) допускают асимптотику:

$$
\lambda_{k}(z)=i \sqrt{\lambda_{k} z}-\frac{1}{2}\left(A_{0} e_{k}, e_{k}\right)+O\left(z^{-1 / 2}\right), \quad z \rightarrow \infty .
$$

А отсюда и из условия 2.2 автоматически следует, что

$$
\left(A e_{k}, e_{k}\right) \geqslant 0, \quad k=1, \ldots, m
$$

Строгие же неравенства (2.5) гарантируют, что с ростом $z$ собственные значения $\lambda_{k}(z), \bar{\lambda}_{k}(z)$ не приближаются к мнимой оси. Отметим, далее, что при строгом нарушении условия 2.2 или 2.3 , когда при некотором $z_{0}>0$ у пучка (2.3) есть собственное значение в полуплоскости $\operatorname{Re} \lambda>0$, нулевое решение краевой задачи $(2.1)$ экспоненциально неустойчиво с показателем экспоненты порядка единищы. Тем самьм, заведомо неустойчивыми будут и все бифурцирующие из него при $\varepsilon>0$ автоколебательные режимы, т.е. интересующая нас проблема теряет смысл.

При сфформулированных ограничениях, следуя методике работ [5]-[7], автоколебания в краевой задаче (2.1) будем искать в виде

$$
u=\varepsilon^{1 / 2} u_{1}(t, s, x)+\varepsilon u_{2}(t, s, x)+\varepsilon^{3 / 2} u_{3}(t, s, x)+\cdots,
$$

где $s=\varepsilon t$

$$
u_{1}=\xi(s, x) a \exp (i t)+\bar{\xi}(s, x) \bar{a} \exp (-i t),
$$

$a$ - собственный вектор из (2.2), $\xi$ - неизвестная комплексная "амплитуда" колебаний, функции $u_{2}, u_{3}, \ldots$ периодичны по $t$ с периодом $2 \pi$.

Подставляя формулы $(2.6),(2.7)$ в уравнение из (2.1) и приравнивая коэффициенты при $\varepsilon$, для $u_{2}$ получаем уравнение вида

$$
\ddot{u}_{2}+A_{0} \dot{u}_{2}+B_{0} u_{2}=|\xi(s, x)|^{2} c_{0}+\xi^{2}(s, x) c_{1} \exp (2 i t)+\bar{\xi}^{2}(s, x) \bar{c}_{1} \exp (-2 i t),
$$


где точка - дифференцирование по $t$, переменные $s, x$ рассматриваются как параметры, а $c_{0}, c_{1}$ - некоторые постоянные векторы, зависящие от квадратичных слагаемых в тейлоровском разложении $f(u, v)$. Отсюда функция $u_{2}$ однозначно определяется в виде

$$
u_{2}=|\xi|^{2} v_{0}+\xi^{2} v_{1} \exp (2 i t)+\bar{\xi}^{2} \bar{v}_{1} \exp (-2 i t)
$$

где

$$
v_{0}=B_{0}^{-1} c_{0}, \quad v_{1}=\left(-4 I+2 i A_{0}+B_{0}\right)^{-1} c_{1} .
$$

Подчеркнем, что обратимость фигурирующих в (2.10) матриц вытекает из условия 2.1.

После приравнивания коэффициентов при $\varepsilon^{3 / 2}$ для $u_{3}$ получаем уравнение, подобное (2.8), но теперь его правая часть $g(t, s, x)$ - сумма первых и третьих гармоник переменной $t$. Поэтому для нахождения $u_{3}$ в том же виде необходимо и достаточно обращение в нуль скалярного произведения коэффициента при $\exp (i t)$ в $g(t, s, x)$ на вектор $b($ см. (2.2)). Приравнивая указанное скалярное произведение к нулю, для определения неизвестной амплитуды $\xi(s, x)$ приходим к краевой задаче

$$
\xi_{s}=\varkappa_{0} \xi_{x x}+\varkappa_{1} \xi+d|\xi|^{2} \xi,\left.\quad \xi\right|_{x=0}=\left.\xi\right|_{x=\pi}=0
$$

в которой $d$ - комплексная ляпуновская величина обыкновенного уравнения, получающегося из (2.1) при $\varepsilon=0$,

$$
\varkappa_{0}=(D a, b), \quad \varkappa_{1}=-i\left(A_{1} a, b\right)-\left(B_{1} a, b\right),
$$

а $\bar{\xi}$ удовлетворяет комплексно-сопряженной задаче. Отметим, что в отличие от исходной системы (2.1) задача (2.11) в силу (2.4), (2.12) является параболической.

Согласно предложенной Ю. С. Колесовым терминологии [5]-[7] краевую задачу (2.11) будем назьвать квазинормальной формой исходной задачи (2.1). Подобное название оправдано тем, что при $D=0$ уравнение из (2.11) представляет собой укороченную нормальную форму соответствующего обыкновенного уравнения (получающегося из (2.1) при $D=0$ ) на экспоненциально орбитально устойчивом двумерном интегральном многообразии. Если же $D \neq 0$, то при выводе задачи (2.11) отбрасываются слагаемые, хотя и малые по порядку относительно $\varepsilon$, но по силе отнюдь не являющиеся подчиненньпи остальньп. Однако, несмотря на это, определенное соответствие между стационарньми режимами краевых задач (2.11) и (2.1) все же имеет место (см. [5]-[7]). Например, справедливо следующее утверждение.

ТЕОРема 2.1. Предположим, что краевая задача (2.11) имеет автомодельный иикл

$$
\xi(s, x)=\xi_{0}(x) \exp \left(i \alpha_{0} s\right), \quad \alpha_{0}=\text { const },
$$

экспоненциально орбитально устойчивый или дихотомичный (в метрике фазового пространства $\stackrel{\circ}{W} \underset{2}{2})$. Тогда найдется такое $\varepsilon_{0}>0$, что при всех $0<\varepsilon \leqslant \varepsilon_{0}$ в исходной задаче (2.1) этому ииклу соответствует периодическое по т решение с асимптотикой (2.6), (2.7), (2.13) и с теми же свойствами устойчивости.

Основной этап доказательства теоремы - исследование системы в вариациях на приближенном цикле - практически без изменений повторяет аналогичные места 
из работ [17], [18], в которых метод квазинормальных форм обоснован для параболического и гиперболического случаев. Поэтому соответствующие выкладки опустим. Отметим только, что здесь сохраняется итоговая оценка вида (1.70). Далее, доказательство существования нужного числа в целом проводится по изложенной в п. 1.2 схеме. Однако здесь возникает один новый и важньй для дальнейшего технический момент, на котором остановимся чуть подробнее.

Напомним, что, как показано в п. 1.2, при наличии в тейлоровском разложении нелинейности $f(u, v)$ квадратичных слагаемых для доказательства существования точного периодического решения с требуемой асимптотикой приближенное решение необходимо знать с точностью до слагаемых порядка $\varepsilon^{2}$ включительно. Таким образом, в нашем случае следует рассмотреть равенство

$$
u=\varepsilon^{1 / 2} u_{1}(\tau, x)+\varepsilon u_{2}(\tau, x)+\varepsilon^{3 / 2} u_{3}(\tau, x)+\varepsilon^{2} u_{4}(\tau, x, \varepsilon),
$$

где $\tau=\left(1+\varepsilon \alpha_{0}\right) t$, а $2 \pi$-периодические по $\tau$ функции $u_{j}, j=1,2,3$, получаются из уже найденных выше коэффициентов ряда (2.6) при $\xi=\xi_{0}(x)$ и при замене $t$ на $\tau$. Что же касается пока неизвестной функции $u_{4}$, то для нее выходит аналогичное (2.8) уравнение

$$
\begin{aligned}
\ddot{u}_{4}+A_{0} \dot{u}_{4}+B_{0} u_{4}= & H_{0}(x)+H_{1}(x) \exp (2 i \tau)+\bar{H}_{1}(x) \exp (-2 i \tau) \\
& +H_{2}(x) \exp (4 i \tau)+\bar{H}_{2}(x) \exp (-4 i \tau)
\end{aligned}
$$

в котором точка - дифференцирование по $\tau$. Заметим, далее, что в отличие от уравнений для $u_{2}$ и $u_{3}$ правая часть $(2.15)$ заведомо не удовлетворяет требуемым граничным условиям. Связано это с тем, что в формирование коэффициентов $H_{0}, H_{1}$ вносит вклад слагаемое $D \frac{\partial^{2} u_{2}}{\partial x^{2}}$. А отсюда и из $(2.9),(2.10)$ заключаем, что в общем случае $H_{j}(0) \neq 0, H_{j}(\pi) \neq 0, j=0,1$.

Для того чтобы исправить положение, представим $u_{4}$ в виде

$$
u_{4}=u_{4,0}(\tau, x)+u_{4,1}(\tau, x, \varepsilon),
$$

где $u_{4,0}-$ решение уравнения $(2.15)$, в правой части которого удержаны только четвертые гармоники, а $u_{4,1}$ - решение краевой задачи

$$
\begin{gathered}
\left(\frac{\partial^{2}}{\partial \tau^{2}}+A_{0} \frac{\partial}{\partial \tau}+B_{0}-\varepsilon D \frac{\partial^{2}}{\partial x^{2}}\right) u_{4,1}=H_{0}(x)+H_{1}(x) \exp (2 i \tau)+\bar{H}_{1}(x) \exp (-2 i \tau) \\
\left.u_{4,1}\right|_{x=0}=\left.u_{4,1}\right|_{x=\pi}=0 .
\end{gathered}
$$

Отметим, что поскольку $H_{2}(0)=H_{2}(\pi)=0$, то и $u_{4,0}$ будет удовлетворять граничным условиям из (2.1). Решение же краевой задачи (2.16) ищем в виде тригонометрического полинома

$$
u_{4,1}=C_{0}(x, \varepsilon)+C_{1}(x, \varepsilon) \exp (2 i \tau)+\bar{C}_{1}(x, \varepsilon) \exp (-2 i \tau) .
$$

В результате для определения коэффициентов $C_{j}, j=0,1$, приходим к сингулярно возмушенным краевьм задачам

$$
\begin{gathered}
\left(B_{0}-\varepsilon D d^{2} / d x^{2}\right) C_{0}=H_{0}(x),\left.\quad C_{0}\right|_{x=0}=\left.C_{0}\right|_{x=\pi}=0 \\
\left(-4 I+2 i A_{0}+B_{0}-\varepsilon D d^{2} / d x^{2}\right) C_{1}=H_{1}(x),\left.\quad C_{1}\right|_{x=0}=\left.C_{1}\right|_{x=\pi}=0
\end{gathered}
$$


Анализ краевых задач (2.17) сушественно опирается на условие 2.2 , из которого вытекают сразу два следствия. Во-первых, это условие гарантирует однозначную разрешимость каждой из них при всех достаточно малых $\varepsilon>0$, в чем нетрудно убедиться, используя метод Фурье по системе функций $\sin k x, k=1,2, \ldots$ Во-вторых, привлекая результаты монографии [19], убеждаемся в справедливости равномерно по $x \in[0, \pi]$ асимптотических представлений

$$
C_{j}(x, \varepsilon)=C_{j}^{0}(x)+\Pi_{j, 1}\left(\sigma_{1}\right)+\Pi_{j, 2}\left(\sigma_{2}\right)+O(\varepsilon), \quad j=0,1
$$

где

$$
\begin{gathered}
C_{0}^{0}(x)=B_{0}^{-1} H_{0}(x), \quad C_{1}^{0}(x)=\left(-4 I+2 i A_{0}+B_{0}\right)^{-1} H_{0}(x) \\
\sigma_{1}=x / \sqrt{\varepsilon}, \quad \sigma_{2}=(\pi-x) / \sqrt{\varepsilon}
\end{gathered}
$$

а так называемые пограничные функции $\Pi_{0, k}(\sigma), \Pi_{1, k}(\sigma), k=1,2,-$ некоторые решения уравнений

$$
B_{0} \Pi_{0, k}-D \Pi_{0, k}^{\prime \prime}=0, \quad\left(-4 I+2 i A_{0}+B_{0}\right) \Pi_{1, k}-D \Pi_{1, k}^{\prime \prime}=0,
$$

удовлетворяющие условиям

$$
\Pi_{j, 1}(0)=-C_{j}^{0}(0), \quad \Pi_{j, 2}(0)=-C_{j}^{0}(\pi), \quad j=0,1
$$

и стремящиеся к нулю при $\sigma \rightarrow+\infty$. Отметим еще, что асимптотические представления (2.18) сохраняются при дифференцировании по $x$, но при этом остатки в них приобретают порядок $\varepsilon^{1 / 2}$.

Из перечисленных свойств решений краевых задач (2.17) (а точнее говоря, из наличия в них пограничных слоев) вытекают оценки

$$
\left\|C_{j}\right\|_{W_{2}^{1}} \leqslant N \varepsilon^{-1 / 4}, \quad j=0,1
$$

Следовательно, при подстановке формулы (2.14) в уравнение из (2.1) и при переносе всех слагаемых из левой части получившегося равенства в правую невязка выйдет величиной порядка малости $\varepsilon^{9 / 4}$ (в метрике $\left.\stackrel{\circ}{W}_{2}^{1}\left([0, \pi] ; \mathbb{R}^{m}\right)\right)$. А это означает, что при реализации описанной в п. 1.2 схемы доказательства равенство (1.60) необходимо заменить на

$$
u=\varepsilon^{1 / 2} u_{1}(\tau, x)+\varepsilon u_{2}(\tau, x)+\varepsilon^{3 / 2} u_{3}(\tau, x)+\varepsilon^{2} u_{4}(\tau, x, \varepsilon)+\varepsilon^{5 / 4} h .
$$

В результате оценка (1.63) сохранится, а в правой части неравенства (1.64) множитель $\varepsilon^{1 / 2}$ заменится на $\varepsilon^{1 / 4}$, что, очевидно, не повлияет на все дальнейшие рассуждения. 
2.2. Скалярный случай. Отдельному рассмотрению подлежит так называемый скалярный случай, когда в уравнении из (2.1) имеем $m=1, D=\sigma^{2}, \sigma>0$. Из условия 2.1 с необходимостью следует, что тогда $A_{0}=0, B_{0}=1$. Полагая, далее, $A_{1}=-1, B_{1}=0$, приходим к аналогичной (1.1) краевой задаче

$$
u_{t t}-\varepsilon u_{t}+u=\varepsilon \sigma^{2} u_{x x}+f\left(u, u_{t}\right),\left.\quad u\right|_{x=0}=\left.u\right|_{x=\pi}=0
$$

В случае краевой задачи (2.19), в отличие от рассмотренной вьше общей ситуации, фигурирующая в уравнении (2.11) ляпуновская величина $d$ вычисляется достаточно просто: используя тейлоровское разложение (1.5), для нее получаем формулу (1.83). Остальные же параметры квазинормальной формы (2.11) теперь задаются равенствами

$$
\varkappa_{0}=-i \sigma^{2} / 2, \quad \varkappa_{1}=1 / 2
$$

И наконец, следует добавить, что для краевой задачи (2.19) условия 2.2 и 2.3 не выполняются. Точнее говоря, здесь мы сталкиваемся с максимальным вырождением: при всех $z \geqslant 0$ корни уравнения $\lambda^{2}+1+z=0$ чисто мнимые и, в частности, квазинормальная форма (2.11) в силу первого равенства (2.20) не является параболической.

Именно максимальное вырождение и служит причиной того, что при наличии в тейлоровском разложении (1.5) квадратичных слагаемых теорема 2.1 для краевой задачи (2.19) перестает быть верной. Действительно, в случае задачи (2.1) требуемую при доказательстве данной теоремы однозначную разрештмость краевых задач (2.17) обеспечивает условие 2.2. В скалярном же случае вторая из этих задач, а именно краевая задача

$$
-\left(\varepsilon \sigma^{2} d^{2} / d x^{2}+3\right) C_{1}=H_{1}(x),\left.\quad C_{1}\right|_{x=0}=\left.C_{1}\right|_{x=\pi}=0
$$

вообще говоря, неразрешима при $\varepsilon=3 /\left(\sigma^{2} k^{2}\right), k=1,2, \ldots$.

Указанная вьше трудность не является чисто технической, а носит принципиальный характер. Связано это с тем, что в случае произвольной нелинейности периодическое решение задачи (2.19) с асимптотикой (2.6), отвечающее фиксированному автомодельному циклу (2.13), существует заведомо не при всех достаточно малых $\varepsilon>0$. В самом деле, из общих соображений ясно, что краевая задача (2.19) наследует динамику задачи (1.1) при $a \rightarrow 0$. Последняя же, напомним, имеет периодическое решение (1.3), (1.4) с фиксированным номером $n$ отнюдь не при всех достаточно малых значениях параметра $a$.

С другой стороны, при условиях (1.72), т.е. при отсутствии квадратичной нелинейности, ситуация иная. Здесь отпадает надобность в определении $u_{4}$ (см. п. 1.2) и, вследствие этого, теорема 2.1 для краевой задачи (2.19) оказывается справедливой (подробности доказательства см. в статьях [9], [18], где даны две различные версии обоснования метода квазинормальных форм в гиперболическом случае).

Обратимся теперь к краевой задаче

$$
u_{t t}-\varepsilon u_{t}+u=\varepsilon \sigma^{2} u_{x x}+f\left(u, u_{t}\right),\left.\quad u_{x}\right|_{x=0}=\left.u_{x}\right|_{x=\pi}=0
$$

и отметим, что для нее теорема 2.1 верна без каких-либо дополнительных ограничений на линейность $f(u, v)$. Объясняется это тем, что здесь в рассмотрении 
краевой задачи (2.21) нет необходимости, так как функция $u_{4}$, определяющаяся непосредственно из уравнения (2.15) (при $A_{0}=0, B_{0}=1$ ), заведомо удовлетворяет требуемьм граничным условиям. Добавим еше, что впервые указанная теорема для задачи (2.22) была анонсирована в заметке [8], а подробное ее доказательство дано в статье [18], результаты которой вошли также в монографию [10].

Подводя итог, отметим, что сформулированные в п. 2.1 ограничения гарантируют справедливость теоремы 2.1 и для краевой задачи, получаюшейся из (2.1) при замене условий Дирихле на произвольные граничные условия типа Штурма-Лиувилля. В скалярном же случае, когда реализуется максимальное вырождение, данная теорема остается в силе лишш при определенном согласовании краевых условий с нелинейностью. Оптимальными в этом плане являются, естественно, условия Неймана, а также условия периодичности $u(t, x) \equiv u(t, x+2 \pi)$, для которых пригодна любая нелинейность. Что же касается граничных условий Дирихле или, скажем, условий $\left.u\right|_{x=0}=\left.u_{x}\right|_{x=\pi}=0$, то для них теорема 2.1 имеет место лишь при отсутствии в тейлоровском разложении (1.5) квадратичных слагаемых или при надлежашей их малости. Достаточно, к примеру, потребовать, чтобы упомянутые слагаемые были величинами порядка $\varepsilon$.

\section{Список литературы}

1. Bumm A. A. Распределенные автоколебательные системы // Журн. технич. физики. 1934. T. 4. № 1. C. 144-157.

2. Мигулин В.В., Медведев В.И., Мустель Е.Р., Парыцин В.Н. Основы теории колебаний. М.: Наука, 1988.

3. Ланда П. С. Нелинейные колебания и волны. М.: Наука, 1997.

4. Колесов А.Ю., Розов Н.Х. Асимптотическая теория колебаний в системе Витта // Совр. матем. и ее прилож. Т. 67. М.: ВИНИТИ, 1999. С. 5-67.

5. Колесов Ю. С. Задача паразит-хозяин // Динамика биологических популяций. Межвуз. сб. Горький: Горьковский ун-т, 1984. С. 28-34.

6. Колесов Ю.С. Об одной бифуркационной теореме в теории автоколебаний распределенных систем // Дифференц. уравнения. 1985. Т. 21. №10. С. 1709-1713.

7. Колесов Ю. С. Метод квазинормальных форм в задаче об установившихся режимах параболических систем с малой диффузией // Укр. матем. журн. 1987. Т. 39. № 1 . C. $27-34$.

8. Колесов А. Ю., Колесов Ю. С. Бифуркация автоколебаний сингулярно возмущенного волнового уравнения // Докл. АН СССР. 1990. Т. 315. № 2. С. 281-283.

9. Колесов Ю.С. Асимптотика и устойчивость нелинейных параметрических колебаний сингулярно возмущенного телеграф̆ного уравнения // Матем. сб. 1995. Т. 186. № 10. C. $57-72$.

10. Колесов А.Ю., Мищенко Е.Ф., Розов Н.Х. Асимптотические методы исследования периодических решений нелинейных гиперболических уравнений // Труды МИАН. 1998. T. 222.

11. Боголюбов H. Н., Митропольский Ю. А. Асимптотические методы в теории нелинейных колебаний. М.: Наука, 1974.

12. Васильева А.Б., Кащенко С.А., Колесов Ю.С., Розов Н.Х. Бифуркация автоколебаний нелинейных параболических уравнений с малой диффузией // Матем. сб. 1986. T. 130. № 4. C. 488-499.

13. Колесов Ю. С., Майоров В. В. Новый метод исследования устойчивости решений линейных дифференциальных уравнений с близкими к постоянным почти периодическими коэффициентами // Дифференц. уравнения. 1974. Т. 10. № 10. С. 1778-1788.

14. Колесов А. Ю. Устойчивость автоколебаний телеграффного уравнения, бифурцирующих из состояния равновесия // Матем. заметки. 1992. Т. 51. № 2. С. 59-65. 
15. Колесов А. Ю. Параметрические колебания решений телеграффного уравнения с умеренно малой диффузией // Сиб. матем. журн. 1992. Т. 33. №6. С. 79-86.

16. Камбулов В. Ф., Колесов А. Ю., Розов Н.X. Существование и устойчивость быстро осциллирующих циклов у нелинейного телеграфного уравнения // ЖВМ и МФ. 1998. T. 38. №8. C. 1287-1300.

17. Колесов Ю. С. Бифуркация инвариантных торов параболических систем с малой диффузией // Матем. сб. 1993. Т. 184. №3. С. 121-136.

18. Камбулов В.Ф., Колесов А. Ю., Розов Н.Х. Бифуркация пространственно неоднородных циклов у нелинейного волнового уравнения с малой диффузией // Труды ММО. 1998. T. 59. C. $197-220$.

19. Васильева А.Б., Бутузов В.. . Асимптотические разложения решений сингулярно возмущенных уравнений. М.: Наука, 1973.

Ярославский государственный университет им. П. Г. Демидова; Московский государственный университет им. М.В. Ломоносова
Поступила в редакцию 26.03.2001 